\title{
Evidence for a Compact Object in the Aftermath of the Extra-Galactic Transient AT2018cow
}

Dheeraj Pasham ( $\nabla$ dheeraj@space.mit.edu )

Massachusetts Inst of Technology

Wynn Ho

Haverford College https://orcid.org/0000-0002-6089-6836

William Alston

ESA

\section{Ronald Remillard}

Massachusetts Inst of Technology https://orcid.org/0000-0003-4815-0481

\section{Mason $\mathrm{Ng}$}

Massachusetts Inst of Technology

Keith Gendreau

NASA GSFC https://orcid.org/0000-0001-7115-2819

Brian Metzger

Columbia University

\section{Diego Altamirano}

Southampton University https://orcid.org/0000-0002-3422-0074

Deepto Chakrabarty

Massachusetts Inst of Technology

\section{Andrew Fabian}

University of Cambridge https://orcid.org/0000-0002-9378-4072

Jon Miller

University of Michigan

Peter Bult

NASA Goddard Space Flight Center

\section{Zaven Arzoumanian}

X-ray Astrophysics Laboratory, NASA Goddard Space Flight Center, Greenbelt, MD 20771

\section{James Steiner}

Center for Astrophysics | Harvard \& Smithsonian

\section{Tod Strohmayer}

NASA/GSFC

Francesco Tombesi

Tor Vergata University of Rome Jeroen Homan 
Eureka Scientific, Inc. https://orcid.org/0000-0001-8371-2713

\section{Edward Cackett}

Wayne State University https://orcid.org/0000-0002-8294-9281

\section{Alice Harding}

Los Alamos National Laboratory

\section{Research Article}

Keywords: Fast Blue Optical Transients (FBOTs), astrophysical phenomena, AT2018cow

Posted Date: May 10th, 2021

DOl: https://doi.org/10.21203/rs.3.rs-465345/v1

License: (c) (i) This work is licensed under a Creative Commons Attribution 4.0 International License. Read Full License

Version of Record: A version of this preprint was published at Nature Astronomy on December 13th, 2021. See the published version at https://doi.org/10.1038/s41550-021-01524-8. 
Evidence for a Compact Object in the Aftermath of the Extra-Galactic Transient AT2018cow 
Dheeraj R. Pasham ${ }^{1 *}$, Wynn $\mathrm{Ho}^{2}$, William Alston ${ }^{3}$, Ronald Remillard ${ }^{1}$, Mason $\mathrm{Ng}^{1}$, Keith Gendreau ${ }^{4}$, Brian Metzger ${ }^{5}$, Diego Altamirano ${ }^{6}$, Deepto Chakrabarty ${ }^{1}$, Andrew Fabian ${ }^{7}$, Jon Miller ${ }^{8}$, Peter Bult ${ }^{4,9}$, Zaven Arzoumanian ${ }^{4}$, James Steiner ${ }^{10}$ Tod Strohmayer $^{4}$, Francesco Tombesi ${ }^{4,9,11,12}$, Jeroen Homan ${ }^{13}$, Edward Cackett ${ }^{14}$, Alice Harding ${ }^{15}$

${ }^{1}$ Kavli Institute for Astrophysics and Space Research, Massachusetts Institute of Technology, Cambridge, MA 02139, USA

${ }^{2}$ Department of Physics and Astronomy, Haverford College, 370 Lancaster Ave, Haverford, PA 19041

${ }^{3}$ European Space Agency (ESA), European Space Astronomy Centre (ESAC), Villanueva de la Cañada, Madrid, E-28691, Spain ${ }^{4}$ NASA Goddard Space Flight Center, Greenbelt, MD, USA

${ }^{5}$ Columbia Astrophysics Laboratory, Columbia University, New York, NY 10027, USA

${ }^{6}$ Department of Physics and astronomy, University of Southampton, University Road, Southampton, SO17 1BJ, UK

${ }^{7}$ Institute of Astronomy, University of Cambridge, UK

${ }^{8}$ Department of Astronomy, 311 West Hall, 1085 South University Ave.,

Ann Arbor, MI 48109-1107

${ }^{9}$ Department of astronomy, University of Maryland College Park, MD, 20742

${ }^{10}$ Center for Astrophysics, Harvard, Cambridge, MA

${ }^{11}$ Department of Physics, Tor Vergata University of Rome, Via della Ricerca Scientifica 1, 00133 Rome, Italy

${ }^{12}$ INAF Astronomical Observatory of Rome, Via Frascati 33, 00040 Monte Porzio Catone, Italy

${ }^{13}$ Eureka Scientific, Inc., Oakland, CA 94602, USA

${ }^{14}$ Department of Physics and astronomy, Wayne State University, 2155 Old Main, 4841 Cass Avenue, Detroit, MI 48201

${ }^{15}$ Theoretical Division, Los Alamos National Laboratory, Los Alamos, NM 87545

*To whom correspondence should be addressed; E-mail: dheeraj@ space.mit.edu

Fast Blue Optical Transients (FBOTs) are mysterious extragalactic explosions that may represent a new class of astrophysical phenomena (1). Their fast 
time to maximum brightness of less than 10 days and decline over less than 2 months and unusual optical spectra and evolution are difficult to explain within the context of core-collapse of massive stars which are powered by radioactive decay of Nickel-56 and evolve slowly on months timescales $(2,3)$. AT2018cow (at a redshift of 0.014 ) is an extreme FBOT both in terms of rapid evolution and high X-ray and bolometric luminosities (4-7). Several alternative hypotheses have been proposed to explain its unusual properties. These include shock interactions with dense circumstellar medium $(5,8,9)$, tidal disruption of a star by a $10^{4-6}$ solar mass black hole $(7,10)$, a failed supernova with fallback accretion onto a newborn black hole (5), neutron star formed in a supernova (11) or from merging compact objects $(12,13)$, etc. Here, we present evidence for a high-amplitude (fractional root-mean-squared amplitude of $>30 \%$ ) quasi-periodic oscillation (QPO) of AT2018cow's soft X-rays with a centroid frequency of roughly $225 \mathrm{~Hz}$ (statistically significant at the $3.7 \sigma$ level, or a false alarm probability of $0.02 \%$ ). This signal is found in the average power density spectrum of data taken over the entire outburst lasting roughly 60 days and thus suggests that the signal is highly persistent over several hundreds of millions of cycles $(60$ days $\times 225 \mathrm{~Hz})$. This high frequency (rapid timescale) of $225 \mathrm{~Hz}(4.4 \mathrm{~ms})$ argues for the presence of a compact object in AT2018cow which can either be a neutron star or a black hole, and disfavors circumstellar medium interactions for the origin of X-ray emission. Also, the QPO's timescale sets an upper limit on the compact object's mass to be 850 solar masses, and thus disfavors models with a heavier black hole. If the QPO represents the spin period of a neutron star we can also set upper limits on its magnetic field under different scenarios. This work highlights a 
new way of using high time-resolution X-ray observations to study FBOTs.

High-cadence sky surveys that can scan the same portions of the sky multiple times per night have uncovered a new class of fast-evolving optical transients $(2,3,14)$. These "fast" transients rise to their peak brightness within $\lesssim 10$ days and fade away within a month or two (e.g., (2)). They are spatially coincident with external galaxies but are offset from their nuclei (e.g., $(2,15))$. Their optical spectra are often blue with occasional presence of Hydrogen and Helium features and are unlike any known class of supernovae (16). Their peak luminosities range from the faint end of core-collapse supernovae to the bright end of superluminous supernovae (see Fig. 1 of (1)). Their fast rise times are difficult to reconcile with an origin from radioactive heating by ${ }^{56} \mathrm{Ni}$ which produces light curves that evolve on much slower timescale of weeks to months (see, for example, Fig. 20 of (2)).

Thus, several alternate mechanisms have been proposed to explain the properties of these luminous Fast, Blue Optical Transients (FBOTs). These include emission from the interaction of the supernova shockwave with a dense circumstellar medium (e.g., (17)), injection of energy from spin down of a young magnetar formed either in a core-collapse supernova or a binary neutron star merger (e.g., $(11,13))$, accretion onto a newly formed compact object in a failed supernova (e.g., (18)), mergers of binary white dwarfs (e.g., (19)), and intermediate-mass black holes (IMBHs: a few $\times 10^{4-5} M_{\odot}$ ) tidally disrupting stars $(7,10)$. Given the diversity in their optical spectra evolution, peak brightness and total energy output, it is likely that they are a heterogeneous population.

Prior to June 2018, all bright FBOTs were first identified in archival images (e.g., (2, 15, 20,21)). AT2018cow, discovered by the ATLAS sky survey (22) in a galaxy at a distance of $\approx 60 \mathrm{Mpc}(6)$, was the first FBOT discovered in real time. Its brightness rise of more than 5.7 magnitudes in just 4 days (see Fig. 1 of (6)) was remarkable and its peak bolometric luminosity of $4 \times 10^{44} \mathrm{erg} \mathrm{s}^{-1}$ makes it the brightest FBOT known so far (1). As the discovery was promptly 
reported (23), the source received a significant amount of multi-wavelength coverage. Radio, millimeter, optical, UV, X-ray and gamma ray properties of the source are described in various papers $(4-7,10)$. However, in spite of exquisite coverage the physical origin of AT2018cow remains elusive.

Given its high X-ray luminosity (peak value of a roughly $10^{43} \mathrm{erg} / \mathrm{s},(5)$ ) and variability on timescales of a few tens of hours (5), compact object (accretion) powered scenarios have been proposed for AT2018cow. These suggestions include emission from tidal disruption of a star by an intermediate-mass black hole with mass in the range of $10^{4-6} M_{\odot}(7)$, fallback accretion in a failed supernova $(5,24)$, and energy injection by a newly born neutron star in a supernova (25).

Several works over the last few decades (26) have found that when accreting compact object (stellar-mass black hole/neutron star) X-ray binaries go into outbursts-due to enhanced accretion-they sometimes exhibit high-frequency $($ a few $\times(10-100) \mathrm{Hz})$ quasi-periodic variability in their X-ray brightness. There is no clear consensus on the exact mechanisms that produce these so-called High-Frequency Quasi-Periodic Oscillations (HFQPOs) but it is generally agreed upon that they originate from a region close to the compact object where the dynamics of motion are dictated by the compact object's strong gravitational field (see (26) and references therein), and they represent a direct evidence for the presence of a compact object. More recently, analogous HFQPOs (frequencies of a few $\mathrm{mHz}$ ) have also been found in stellar tidal disruption events involving $\sim 10^{6} M_{\odot}$ black holes (27-29) suggesting that, perhaps, such QPOs are universal among all compact object systems that undergo extreme changes in accretion.

To test these hypotheses for a compact object (accretion) powered scenario, we studied AT2018cow's X-ray $(0.25-2.5 \mathrm{keV})$ variability using an average power density spectrum derived from the entire monitoring data taken by the Neutron Star Interior Composition Explorer (NICER) on board the International Space Station. We find evidence for an X-ray QPO in the average PDS (left panel of Fig. 1). The QPO signal has a centroid frequency, full width half 
maximum (FWHM) and a fractional root-mean-squared amplitude of $224.4 \pm 1.0 \mathrm{~Hz},<16 \mathrm{~Hz}$, and $30 \pm 3 \%$, respectively. Using a rigorous Monte Carlo approach (see supplementary material, SM) that takes into account the nature of the underlying noise continuum and the search trials, we find the global false alarm probability of this signal to be $\approx 2 \times 10^{-4}$ (or $3.7 \sigma$ equivalent for a normal distribution; see right panel of Fig. 1 and SM).

We rule out various instrumental and particle backgrounds as the origin for this QPO signal (See SM). Based on the long term light curves derived from NICER and Neil Gehrels Swift X-ray telescope, which has imaging capability (see top panel of Fig. 2), and late time $X M M$ Newton X-ray images of AT2018cow's field of view, we also rule out a contaminating source as the origin of this QPO (see Fig. 3). Furthermore, over the last three years of NICER operations a signal of similar nature has never been found in any of the several dozens of other targets (see, for example, Fig. S8). Based on these tests, we conclude that the signal is consistent with originating from AT2018cow.

After establishing that the signal is statistically acceptable and ruling out an instrumental and a background origin, we extracted the QPO's signal-to-noise as a function of the accumulated exposure (See Fig. S7 and supplementary movie S1). It is evident that the QPO's strength increases gradually with increasing exposure. This suggests that the signal is persistent and present at some level throughout the $\sim 2$ month monitoring period. Taken at face value, this suggests that it is stable over $\sim 60$ days $/ 4.44 \mathrm{~ms} \gtrsim 10^{9}$ cycles. Interestingly, the mean slope of the curve in Fig. S7 also steepens around the same time (near day 17) when high-amplitude X-ray flares start to appear on days timescale (see the blue diamonds in the upper panel of Fig. 2). Moreover, the fractional rms of the QPO jumps around day 17 which also coincides with the time when the optical spectrum of AT2018cow underwent dramatic changes (see (5) for details). We separated the total exposure into two epochs, before and after day 17 . We extract an average PDS from each of these two time intervals and the QPO is fit with a Lorentzian. The 
QPO's fractional rms amplitude appears to be higher at later times (see bottom panel of Fig. 2).

The frequency of this QPO alone can set stringent constraints on the underlying physical mechanism producing X-rays in AT2018cow. The causality argument suggests that the physical size of an object producing this signal cannot be larger than the light crossing size, i.e., speed of light $\times(1 / 225 \mathrm{~s}) \approx 1.3 \times 10^{8} \mathrm{~cm}$. This small size points us naturally towards a compact object and disfavors emission from shock interactions $(5,8)$. If that compact object is a black hole then assuming the emission originates from the innermost stable circular orbit allows us to set an upper limit on the black hole mass. These limits are $95 M_{\odot}$ and $850 M_{\odot}$ for a maximally prograde spinning (spin has the same direction as the material falling in) and a retrograde (vice versa) spinning black hole, respectively. A larger emission radius (in units of gravitational radii) would require the object to be even more compact. This rules out a heavy IMBH ( $\gtrsim 850 M_{\odot}$ ) in AT2018cow (10).

$\mathrm{HFQPOs}$ with frequencies in the hundreds of $\mathrm{Hz}$ range have been seen in a handful of stellar-mass black holes with known dynamical mass estimates (26). They often appear in pairs with frequency ratio of 2:3 (26). In these systems it appears that the black hole mass scales inversely with the HFQPO frequency (see, for example, Fig. 4.17 of (26)). While the frequency, coherence and width of AT2018cow's QPO are similar to HFQPOs of stellar-mass black holes, the observed fractional rms amplitude is high (25-45\% compared to a few percent in stellar-mass black holes (26)), and a harmonic is not apparent here. Furthermore, the QPO's energy dependence is also uncertain given our narrow X-ray band pass of $0.25-2.5 \mathrm{keV}$. Also, the absence of any red noise (even at lower frequencies, i.e., a few $\times \mathrm{mHz}$; see Fig. S3) is unlike accreting X-ray binaries. All the stellar-mass X-ray binaries with HFQPOs are relatively highly inclined (26). Given AT2018cow's high (a few $\times 10^{42} \mathrm{erg} / \mathrm{s}$ ) average X-ray luminosity beaming is likely present, and this points towards a low inclination for this system. Thus, given all these factors, it is unclear if a direct comparison can be made with HFQPOs of stellar-mass black 
hole binaries. Nevertheless, if we assume the same scaling law applies and that $225 \mathrm{~Hz}$ is the fundamental harmonic, then the implied black hole mass in AT2018cow is $\sim 4 M_{\odot}$.

Alternatively, the compact object could be a neutron star with the QPO representing its spin rate of $225 \mathrm{~Hz}$. Since the QPO has a width of $<16 \mathrm{~Hz}$, we can constrain a change in the spin rate during the 60 days of observation to be $<16 \mathrm{~Hz} / 60 \mathrm{~d}=3 \times 10^{-6} \mathrm{~Hz} \mathrm{~s}^{-1}$. The standard scenario for spin rate evolution of a non-accreting/isolated neutron star then implies an upper limit on the stellar magnetic field of $\sim 2 \times 10^{13} \mathrm{G}$, and qualitatively similar constraints on magnetic field can also be set by considering, for example, evolution of the X-ray luminosity or accretion (see Section 3 in SM). The frequency and X-ray luminosity of the QPO are consistent with a young pulsar with a Crab-like magnetic field of a few times $10^{12} \mathrm{G}$ (depending on X-ray efficiency). Such a scenario cannot however explain the high luminosity during the early phases of AT2018cow, unless it underwent large magnetic field strength or orientation changes. The observed rapid luminosity drop after roughly 40 days (see Fig. 2 and also Fig. 4 of (5)) would also require changes, perhaps in the surrounding material leading to the loss of a clear line of sight to the pulsar or its X-ray reflections.

Within the bounds of a central engine being either a neutron star, stellar-mass black hole or a few hundred $\mathrm{M}_{\odot}$ black hole, the QPO presented here can be produced under different scenarios. For example, it has been proposed that a flare like AT2018cow could arise from young star clusters containing stellar-mass black holes (mass $\sim 10 \mathrm{M}_{\odot}$ ) tidally disrupting a main sequence star (30) or from fallback accretion of material in a failed supernova whose core collapses into either a neutron star or a stellar-mass black hole (7). In all these scenarios, such a QPO signal could be envisioned. The detection of a star cluster near or coincident with AT2018cow could help to differentiate between these models. The implications of this QPO for AT2018cow's existing models are summarized in Table 1.

In summary, we present evidence for a $225 \mathrm{~Hz}$ X-ray QPO persistent for billions of cycles 
from AT2018cow statistically significant at the $3.7 \sigma$ level. Previous works (5) have suggested a compact object in AT2018cow based on X-ray variability on a few tens of hours timescale and also its X-ray spectral resemblance to some accreting systems. The detection of regular variations on millisecond timescale presented here provides the most direct way to infer the presence of a compact object, although it still remains unclear whether that compact object is a stellar-mass black hole or a neutron star. If AT2018cow originated from the death of a massive star, our findings represent the birth of a compact object in a supernova. Similar signals in future FBOTs could allow astronomers to study infant compact objects immediately after birth. 
Table 1: Models for AT2018cow's X-ray emission and their validity against the $225 \mathrm{~Hz}$ QPO reported here. This table can be considered as a modified extension of Table 2 of (5).

\begin{tabular}{|c|c|c|c|}
\hline Model/class of models & References $^{\bullet}$ & $\begin{array}{c}\text { Consistent } \\
\text { with QPO? }\end{array}$ & Notes \\
\hline $\begin{array}{l}\text { Shock interactions with } \\
\text { circumstellar medium } \\
\text { (CSM) }\end{array}$ & $(4,8)$ & No & $\begin{array}{l}\text { CSM interaction could explain emission at other } \\
\text { (non X-ray) wavelengths, i.e., optical and radio but } \\
\text { inconsistent with rapid X-ray variability }\end{array}$ \\
\hline $\begin{array}{l}\text { An embedded internal } \\
\text { shock formed from } \\
\text { interaction with dense CSM }\end{array}$ & (5) & No & $\begin{array}{l}\text { A compact embedded internal shock is, in } \\
\text { principle, consistent with the size constraint } \\
\text { provided by the } 225 \mathrm{~Hz} \text { QPO. However, all X-ray } \\
\text { QPOs known thus far in literature are from compact } \\
\text { objects. So an internal shock model is disfavored. }\end{array}$ \\
\hline $\begin{array}{c}\text { Accreting } \\
\text { intermediate-mass black } \\
\text { hole }\left(\gtrsim 10^{3} M_{\odot}\right)\end{array}$ & $(7,10,31)$ & No & $\begin{array}{l}\text { Based on causality argument the compact object } \\
\text { producing the QPO has to be less than } 850 M_{\odot} \text { (see } \\
\text { main text) }\end{array}$ \\
\hline $\begin{array}{l}\text { Neutron star (formed from } \\
\text { merging white } \\
\text { dwarfs/Supernova) }\end{array}$ & $(6,12)$ & Yes & $\begin{array}{l}\text { Constraints on magnetic field if the } 225 \mathrm{~Hz} \text { QPO } \\
\text { represents the spin period. However, the } \\
\text { persistence of the signal in a narrow frequency } \\
\text { range is challenging to explain (see SM) }\end{array}$ \\
\hline $\begin{array}{c}\text { Stellar-mass black hole } \\
\text { (accreting from outer layers } \\
\text { of a failed supernova/tidally } \\
\quad \text { disrupting a star) }\end{array}$ & $(7,30,32,33)$ & Yes & $\begin{array}{l}\text { The QPO frequency is similar to those often seen in } \\
\text { known stellar-mass black holes but the X-ray } \\
\text { luminosity, rms, and QPO's stability are unlike any } \\
\text { known stellar-mass black hole systems (see main } \\
\text { text) }\end{array}$ \\
\hline
\end{tabular}



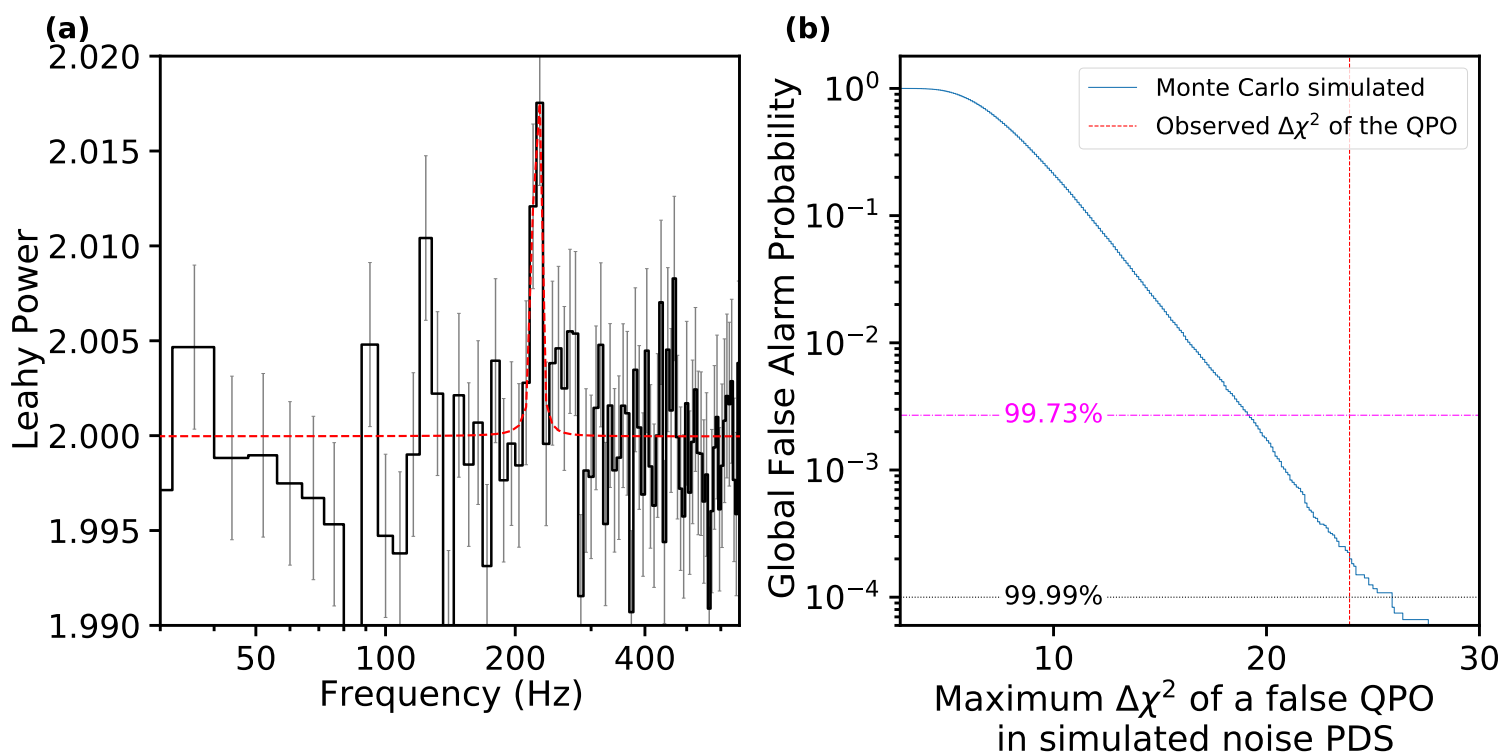

Figure 1: (a) Average X-ray PDS of AT2018cow showing evidence for a quasi-periodicity near $225 \mathrm{~Hz}$. This PDS was computed by averaging 105 256-second soft X-ray (0.25-2.5 $\mathrm{keV}$ ) light curves sampled at $1 / 2048 \mathrm{~s}$. The resulting PDS was further re-binned by a factor of 2048 which gives a frequency resolution of $8 \mathrm{~Hz}$. The strongest excess above the Poisson noise level of 2 is around $225 \mathrm{~Hz}$. The power values in the rest of the PDS continuum are consistent with white noise (see SM sections 2.2.2 and 2.2 and Figures S2, S1, S3). The PDS is normalized such that the mean value surrounding $225 \mathrm{~Hz}$ is equal to the Poisson value of 2 . The best-fit constant + Lorentzian models are indicated by dashed red curves. (b) Estimates for the statistical significance of the $225 \mathrm{~Hz}$ QPO. The likelihood of finding a QPO from noise fluctuations (y-axis) vs the maximum improvement in $\chi^{2}$ by fitting the simulated noise PDS with a constant+Lorentzian over modeling it with a constant at every frequency searched during the identification of the signal on the left (see section 2.2.5 for more details). The global false alarm probability of finding a QPO as strong as the one observed is $\approx 2 \times 10^{-4}$ ( 1 in 5000; $\approx 3.7 \sigma$ ). 


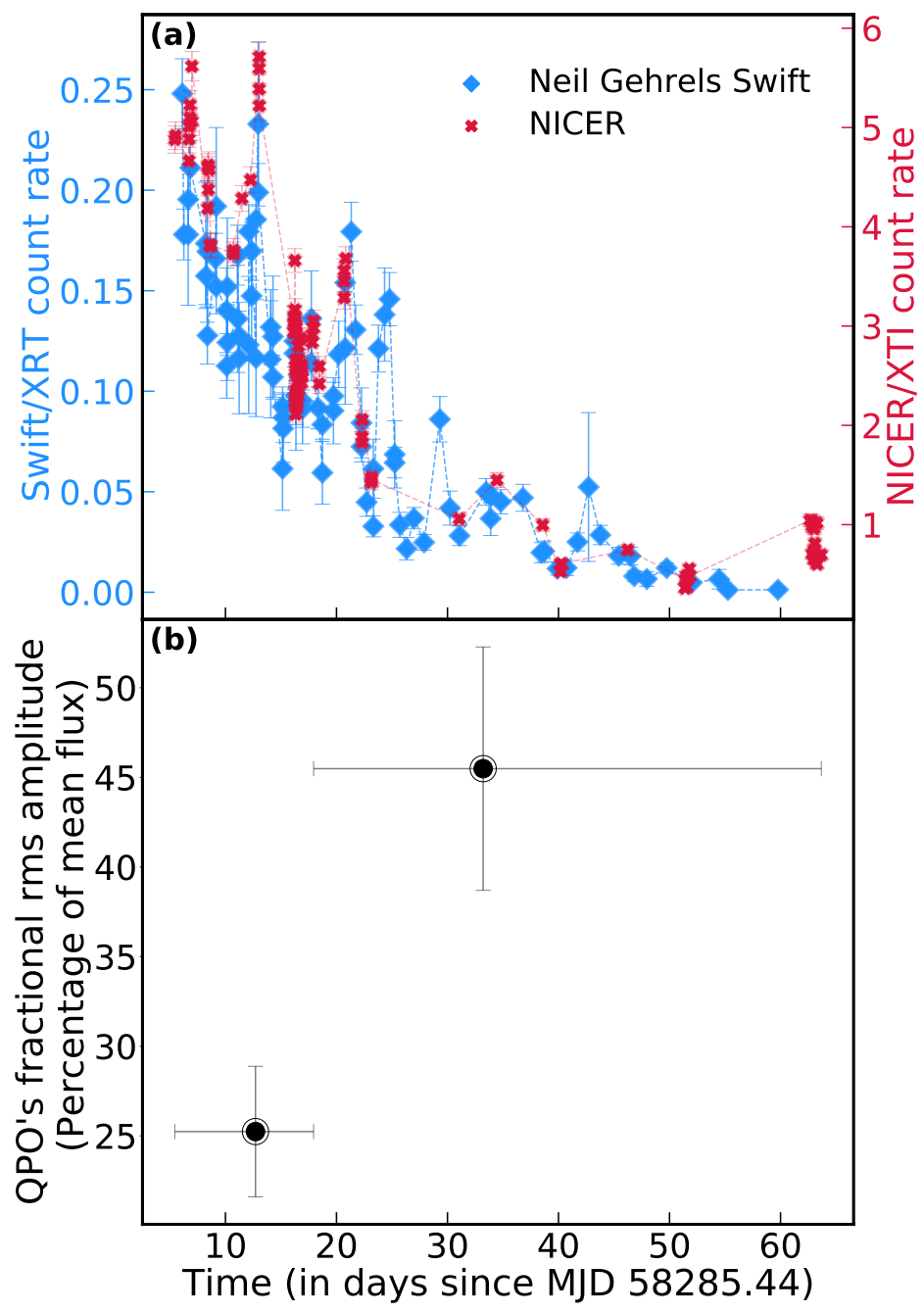

Figure 2: (a) Comparison of NICER/XTI and Neil Gehrels Swift/XRT long-term light curves. Both y-axes are in units of counts/sec. It is evident that AT2018cow's long-term soft $\mathrm{X}$-ray $(0.25-2.5 \mathrm{keV})$ variability as observed by the non-imaging NICER telescope is same as that observed with Neil Gehrels Swift/XRT (0.3-2.5 keV) which has imaging capability. This suggests that the flux observed by NICER is dominated by AT2018cow with minimal contamination from other nearby astrophysical sources. (b) Fractional root-mean-squared amplitude of the $225 \mathrm{~Hz}$ QPO vs time. The signal appears to be stronger during the end of the outburst. The two values are $25 \pm 4$ and $45 \pm 7 \%$ corresponding to exposures during $12.7_{-7.3}^{+5.2}$ and $33.2_{-15.3}^{+30.5}$ days, respectively. The jump in the QPO's strength coincides with higher levels of variability on days timescale (XRT light curve in (a)) and also with the dramatic changes in optical spectra seen around the same time (5). 


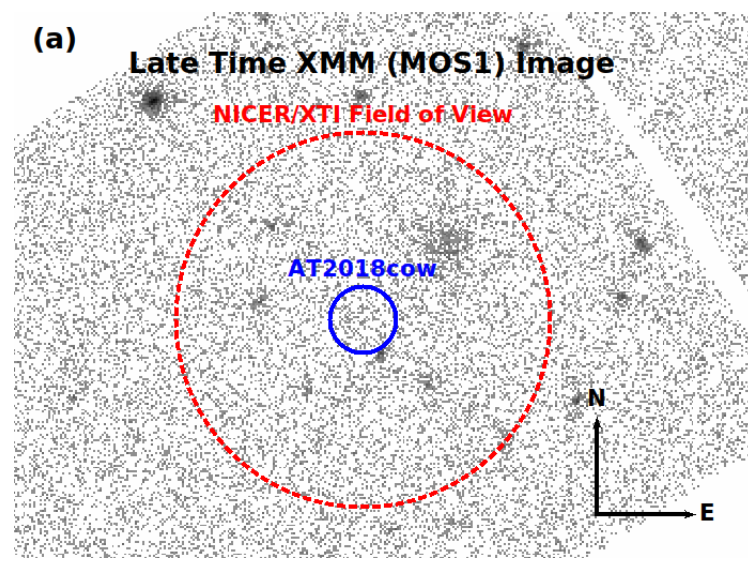

(b)

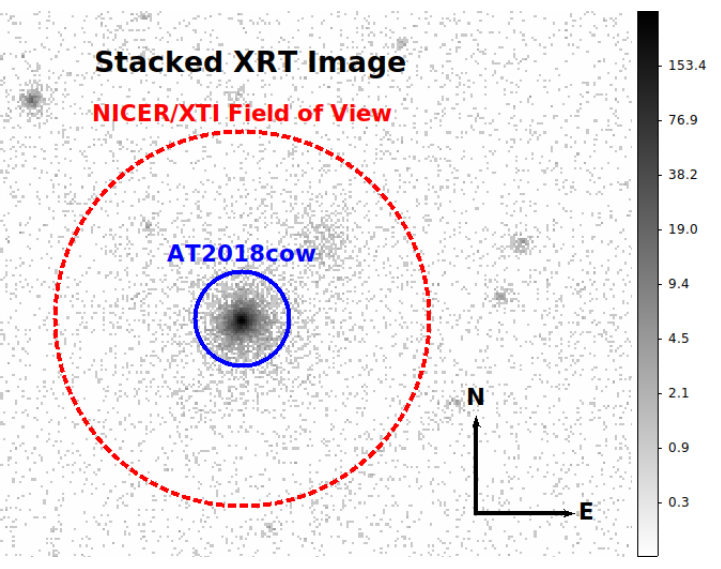

Figure 3: XMM-Newton and Swift images of NICER's field of view of AT2018cow showing that there was minimal contamination from field sources. (a) XMM-Newton/MOS1 image of AT2018cow's field of view long after AT2018cow faded. The position of AT2018cow is indicated by a blue circle with a radius of 33". There is no contaminating point source directly coincident with AT2018cow. (b) Stacked Neil Gehrels Swift/XRT image of the field of view of AT2018cow. The blue circle of radius 47" is centered on AT2018cow's optical position $(16: 16: 00.220+22: 16: 04.91 ; \mathrm{J} 2000.0)$. This particular image was extracted by using all the archival XRT images as of July 2020. It is evident that while there are a few point sources present in NICER's field of view, their contribution to overall XTI flux is negligible when compared to AT2018cow (see also Fig. 2). In both panels the outer/red (dashed) circles show NICER/XTI's approximate field of view of 3.1'. The north and east arrows are each 100" long. The colorbar show counts. 


\section{References}

1. C. Inserra, Nature Astronomy 3, 697 (2019).

2. M. R. Drout, et al., Astrophys. J. 794, 23 (2014).

3. M. Pursiainen, et al., Mon. Not. R. Astron. Soc. 481, 894 (2018).

4. L. E. Rivera Sandoval, et al., Mon. Not. R. Astron. Soc. 480, L146 (2018).

5. R. Margutti, et al., Astrophys. J. 872, 18 (2019).

6. S. J. Prentice, et al., Astrophys. J. 865, L3 (2018).

7. D. A. Perley, et al., Mon. Not. R. Astron. Soc. 484, 1031 (2019).

8. S.-C. Leung, et al., Astrophys. J. 903, 66 (2020).

9. O. D. Fox, N. Smith, Mon. Not. R. Astron. Soc. 488, 3772 (2019).

10. N. P. M. Kuin, et al., Mon. Not. R. Astron. Soc. 487, 2505 (2019).

11. D. Kasen, L. Bildsten, Astrophys. J. 717, 245 (2010).

12. M. Lyutikov, S. Toonen, Mon. Not. R. Astron. Soc. 487, 5618 (2019).

13. B. D. Metzger, A. L. Piro, Mon. Not. R. Astron. Soc. 439, 3916 (2014).

14. M. Tanaka, et al., Astrophys. J. 819, 5 (2016).

15. A. Rest, et al., Nature Astronomy 2, 307 (2018).

16. A. V. Filippenko, $A R A \& A$ 35, 309 (1997).

17. R. A. Chevalier, C. M. Irwin, Astrophys. J. 747, L17 (2012). 
18. K. Kashiyama, E. Quataert, Mon. Not. R. Astron. Soc. 451, 2656 (2015).

19. J. Brooks, et al., Astrophys. J. 850, 127 (2017).

20. M. Tanaka, et al., Astrophys. J. 819, 5 (2016).

21. M. Pursiainen, et al., Mon. Not. R. Astron. Soc. 481, 894 (2018).

22. J. L. Tonry, et al., Publ. Astron. Soc. Pac. 130, 064505 (2018).

23. S. J. Smartt, et al., The Astronomer's Telegram 11727, 1 (2018).

24. E. Quataert, D. Kasen, Mon. Not. R. Astron. Soc. 419, L1 (2012).

25. P. Mohan, T. An, J. Yang, Astrophys. J. 888, L24 (2020).

26. J. E. McClintock, R. A. Remillard, Black hole binaries (2006), vol. 39, pp. 157-213.

27. D. R. Pasham, et al., Science 363, 531 (2019).

28. R. C. Reis, et al., Science 337, 949 (2012).

29. D. Lin, J. A. Irwin, O. Godet, N. A. Webb, D. Barret, Astrophys. J. 776, L10 (2013).

30. K. Kremer, et al., arXiv e-prints p. arXiv:2012.02796 (2020).

31. J.-H. Chen, R.-F. Shen, Astrophys. J. 867, 20 (2018).

32. J. Dexter, D. Kasen, Astrophys. J. 772, 30 (2013).

33. E. Quataert, D. Lecoanet, E. R. Coughlin, Mon. Not. R. Astron. Soc. 485, L83 (2019).

34. K. C. Gendreau, et al., SPIE (2016), vol. 9905 of Society of Photo-Optical Instrumentation Engineers (SPIE) Conference Series, p. 99051H. 
35. N. Gehrels, et al., Astrophys. J. 611, 1005 (2004).

36. D. N. Burrows, et al., Space Sci. Rev. 120, 165 (2005).

37. F. Jansen, et al., Astron. Astrophys. 365, L1 (2001).

38. L. Strüder, et al., Astron. Astrophys. 365, L18 (2001).

39. G. Prigozhin, et al., SPIE (2012), vol. 8453 of Society of Photo-Optical Instrumentation Engineers (SPIE) Conference Series, p. 845318.

40. M. van der Klis, Timing Neutron Stars (1988), vol. 262, pp. 27-70.

41. S. Vaughan, R. Edelson, R. S. Warwick, P. Uttley, Mon. Not. R. Astron. Soc. 345, 1271 (2003).

42. S. L. Shapiro, S. A. Teukolsky, Black holes, white dwarfs, and neutron stars : the physics of compact objects (1983).

43. B. D. Metzger, P. Beniamini, D. Giannios, Astrophys. J. 857, 95 (2018).

44. V. F. Shvartsman, Soviet Astronomy 15, 342 (1971).

45. A. F. Illarionov, R. A. Sunyaev, Astron. Astrophys. 39, 185 (1975).

46. V. M. Lipunov, Astrophysics of Neutron Stars (1992).

47. K. Davidson, J. P. Ostriker, Astrophys. J. 179, 585 (1973).

48. A. L. Piro, C. D. Ott, Astrophys. J. 736, 108 (2011).

49. K. Parfrey, A. Spitkovsky, A. M. Beloborodov, Astrophys. J. 822, 33 (2016).

50. M. A. Alpar, Astrophys. J. 554, 1245 (2001). 
51. S. M. Ransom, S. S. Eikenberry, J. Middleditch, Astron. J. 124, 1788 (2002).

52. B. C. Andersen, S. M. Ransom, Astrophys. J. 863, L13 (2018).

53. M. Bagchi, D. R. Lorimer, S. Wolfe, Mon. Not. R. Astron. Soc. 432, 1303 (2013). 


\section{Supplementary Materials.}

Materials and Methods

Figs. S1 to S8

Caption for movie $\mathrm{S} 1$

Movie S1

Supplementary Text

All the codes to download the data from HEASARC public archive, reduce it, and reproduce the results in this paper are available as supplementary files 


\section{${ }_{21}$ Supplementary Material.}

\footnotetext{
*Throughout this paper we refer times with respect to this optical discovery date

†https://heasarc.gsfc.nasa.gov/cgi-bin/w3Browse/w3browse.pl
} 


\footnotetext{
${ }^{\ddagger}$ https://www.swift.ac.uk/analysis/xrt/pileup.php
} files.

\subsection{Neil Gehrels Swift/XRT:} energy range.

\subsection{XMM-Newton:}

considered X-ray events in the energy range of 0.25 to $2.5 \mathrm{keV}$. This background cannot be directly subtracted from the light curves, and thus acts to increase the noise in the power density spectra of the source. These are tested in detail in section 2.3. Our entire download and reduction procedure can be easily replicated by running the codes that are available in supplementary

Neil Gehrels Swift started monitoring AT2018cow on MJD 58288.44, roughly 3 days after its optical discovery. The observing cadence varied over the $58 \mathrm{~d}$ window coincident with the NICER observing campaign, i.e., until MJD 58349. For the first $\approx 3$ weeks, Neil Gehrels Swift observed AT2018cow 3-4 times per day and thereafter the cadence was reduced to roughly one exposure per day. The individual exposure duration varied between 200 and $2000 \mathrm{~s}$. In this work we only used XRT data in the band pass of 0.3-2.5 keV to be consistent with NICER's

We started our analysis with the publicly available, raw, level-1 data from HEASARC archive and reprocessed them with the xrtpipeline task of HEASoft. Initially, when the source was bright, data was taken in both the Windowed Timing (WT) and the Photon Counting (PC) modes. But as the source count rate dropped observations were only carried out in the PC mode. For this work, we only used the PC mode data with event grades between 0 and 12 . Source events were extracted from an annular region centered on AT2018cow with the outer radius fixed at 47". This outer radius of 47" corresponds to roughly $90 \%$ (at $1.5 \mathrm{keV}$ ) of the light from a point source (as estimated from XRT's fractional encircled energy function). The inner radius was determined independently for each exposure by accounting for pile-up using the formalism described in the XRT user guide ${ }^{\ddagger}$. Background events were extracted from an annular region centered on AT2018cow with an inner and outer radii of 150" and 210", respectively. These values were chosen to avoid any point sources in that background annulus.

XMM-Newton observed AT2018cow on three separate occasions roughly 37 (obsID: 0822580401; $33 \mathrm{ks}$ ), 82 (obsID: 0822580501; $45 \mathrm{ks}$ ), and 218 days (obsID: 0822580601; 56 ks) after its optical discovery. In this work, we only used the European Photon Imaging Camera (EPIC) data from the first and the last XMM-Newton epochs. The first exposure coincided with the NICER monitoring campaign while the last one was taken several months after AT2018cow faded away. As the data were taken in the full frame mode the Nyquist frequencies of the pn and MOS detectors were $6.8 \mathrm{~Hz}$ and roughly $0.2 \mathrm{~Hz}$, respectively. To constrain the nature of variability on frequencies of a few $\mathrm{Hz}$ we only used pn data from the first dataset. For the last exposure MOS1 provides the best spatial resolution and hence we used only MOS1 data. 
We started XMM-Newton/EPIC data reduction with the raw Observation Data Files (ODF) and reprocessed them using the XMM Science Analysis Software's (xmmsas version 17.0.0) tasks epproc and emproc for the pn and MOS data, respectively. We employed standard data filters of $(P A T T E R N \lesssim 12)$ and $(P A T T E R N \leqslant 4)$ for the pn and the MOS data, respectively. We only considered events in the soft X-ray band of $0.25-2.5 \mathrm{keV}$ to be consistent with NICER's data (see section 1.1). We removed intervals of background flaring by manually inspecting the 10-12 keV light curve as outlined in the XMM-Newton data analysis guide. The source count rates were extracted from a circular region of radius 33" which corresponds to $90 \%$ of the light from a point source as estimated by the fractional encircled energy of EPIC instruments. Background events were extracted from two nearby circular regions of radius 50".

\section{Analysis}

\subsection{NICER identifies a QPO candidate}

We first divided the NICER data into 256-s continuous segments and extracted their light curves with a time resolution of $1 / 2048 \mathrm{~s}$. This resulted in a total of 105 light curve segments, i.e., a cumulative exposure time of 26,880 s. With the mean count rate varying between 5.7 and 0.4 count $\mathrm{s}^{-1}$ this choice of $256 \mathrm{~s}$ ensured $\gtrsim 100$ counts in each segment. A Leahy normalized (Poisson noise level of 2) power density spectrum (PDS) was extracted from each of these individual light curves and they were all combined to obtain an average PDS (see the left panel of Fig. 1). The PDS is consistent with a value of 2 (Poisson noise level) at all frequencies except for excess around $225 \mathrm{~Hz}$.

\subsection{Estimating the global statistical significance}

To estimate the global false alarm probability of this QPO candidate near $225 \mathrm{~Hz}$ by properly accounting for the underlying noise and also all the search trials we devised a Monte Carlo approach. The basic idea is to simulate a large number $\left(\sim 10^{5}\right.$ in our case $)$ of random realizations of the underlying noise process, i.e., light curves that capture the underlying PDS continuum. Then, extract a power spectrum just like the real data, perform a global search (at all frequencies) for QPOs, and finally, estimate the probability of seeing a noise fluctuation that resembles a QPO as strong as the one found in the real data (left panel of Fig. 1). Because a robust significance estimate relies on accurate knowledge of the underlying noise process, at first, we lay extra emphasis on understanding the nature of the PDS continuum.

\subsubsection{Probability plot: a qualitative assessment of noise}

Visually, the power spectrum in Fig. 1 appears to be flat (or white) between a few $\mathrm{Hz}$ to 1024 $\mathrm{Hz}$, except for the bins surrounding the QPO feature at $225 \mathrm{~Hz}$. Formally, a test for flatness or "whiteness" of a power spectrum is a test for whether the power spectral values are $\chi^{2}$ 
distributed (40). First, we assess this qualitatively by extracting a so-called probability plot. It shows the theoretical quantiles of an assumed distribution $\left(\chi^{2}\right.$ with $2 \times 105 \times 2048$ degrees of freedom (dof) scaled by a factor of $1 /(105 \times 2048)$ in the present case) against the ordered sample values, i.e., observed noise powers. This particular $\chi^{2}$ distribution was used to compare because the PDS in the left panel of Fig. 1 was obtained by averaging 105 individual PDS followed by further averaging in frequency by a factor of 2048. If the observed data fall on a straight line on the probability plot then that indicates that they are consistent with the theoretical distribution that they are compared against. To remove the bias from the QPO we removed bins whose frequencies fall between 200 and $250 \mathrm{~Hz}$. Also because we are really only interested in the nature of the noise continuum in the vicinity of $225 \mathrm{~Hz}$ we removed bins with frequencies above $600 \mathrm{~Hz}$. Because there are many bins between 600 and $1024 \mathrm{~Hz}$ they could, in principle, skew the results. It is evident from Fig. S1 that the data points on the probability plot roughly follow a straight line (red line) and thus appear "qualitatively" consistent with a $\chi^{2}$ distribution with $2 \times 105 \times 2048$ dof scaled by a factor of $1 /(105 \times 2048)$. For completeness, we repeated this by considering all bins between $1 / 256 \mathrm{~Hz}$ and $1024 \mathrm{~Hz}$ and the result is the same.

\subsubsection{PDS continuum is consistent with White noise}

To investigate the nature of the PDS continuum quantitatively, we performed the KolmogorovSmirnov (K-S) and the Anderson-Darling goodness-of-fit tests under the null hypothesis that the PDS continuum is white, i.e., the power values between 1/256 and $600 \mathrm{~Hz}$, except for bins between $200-250 \mathrm{~Hz}$, are $\chi^{2}$ distributed distributed with $2 \times 105 \times 2048$ dof scaled by a factor of $1 /(105 \times 2048)$. The basic idea with these statistics is that they measure the maximum deviation between the Empirical Distribution Function (EDF) of the data and that of a comparison distribution. Therefore, the better the distribution fits the data, the smaller these statistics will be.

Before we evaluated the K-S and Anderson-Darling test statistics we computed the EDF and the probability density function (PDF) of the continuum noise powers. These are shown in the top two panels of Figure S2) along with the expected EDF and PDF curves for a $\chi^{2}$ distribution (solid red). Similar to the probability plot, the data appear to track the expected $\chi^{2}$ distribution quite well.

We computed the K-S statistic using the EDF. To evaluate whether this value can be used to reject or not reject the null hypothesis, we calculated the distribution of K-S statistic values of EDFs drawn from the expected distribution: $\chi^{2}$ with $2 \times 105 \times 2048$ dof scaled by a factor of $1 /(105 \times 2048)$. We compute this K-S statistic distribution as follows.

1. First, we randomly draw 57 values from a $\chi^{2}$ with $2 \times 105 \times 2048$ dof. Here, 57 refers to the total number of power spectral continuum values between 100 and $600 \mathrm{~Hz}$ and excluding those with frequencies between 200 and $250 \mathrm{~Hz}$.

2. Then we evaluate the EDF of this random sample of 57 and scale it by $1 /(105 \times 2048)$

3. Finally, we compute its K-S statistic value. 
The above steps are repeated 10,000 times to get a distribution of the K-S test statistic for a $\chi^{2}$ distribution with $2 \times 105 \times 2048$ dof for a given sample size of 57 . This is shown as a blue histogram in the bottom left panel of Fig. S2. AT2018cow's observed K-S test statistic (dashed vertical red line), which is a measure of maximum deviation between the observed EDF and the theoretical Cumulative Distribution Function (CDF), is lower than the typical value (solid magenta vertical line). This indicates that the null hypothesis cannot be rejected even at the $90 \%$ confidence level and suggests that noise powers in the PDS continuum are very much consistent with the expected $\chi^{2}$ distribution, i.e., NICER's PDS continuum is consistent with being white between $1 / 256$ and $1024 \mathrm{~Hz}$.

To ensure the above conclusion is not dependent on the choice of the statistic used we also computed the Anderson-Darling statistic. Similar to above, we computed its distribution using bootstrap simulations (see the bottom right panel of Fig. S2). Again, it is clear that the statistic computed from the observed PDS of AT2018cow (vertical dashed red line) is consistent with the expected $\chi^{2}$ distribution. We repeated all the above tests by considering all the bins between $1 / 256$ to the Nyquist frequency of $1024 \mathrm{~Hz}$ (excluding those between 200-250 Hz) and the results remained the same. We also varied the frequency upper limit from $400 \mathrm{~Hz}$ to $800 \mathrm{~Hz}$ and they all yield the same result.

\subsubsection{Modeling the PDS}

Next, we carried out a straightforward method to test for flatness of the PDS continuum. We fit the continuum, i.e., excluding the bins between 200 and $250 \mathrm{~Hz}$, with two different models: a constant (white noise) and a constant + power-law (white + red noise). The model consisting of just a constant gave a $\chi^{2}$ of 129.9 with 121 dof while the constant+power-law yields a $\chi^{2}$ of 125.1 with 119 dof. We repeated this exercise with a PDS frequency resolution of $1 / 256 \mathrm{~Hz}$, i.e., lowest frequency of $1 / 256 \mathrm{~Hz}$. This also did not yield in significant $\chi^{2}$ improvement. This argues that a power-law component is not formally required by the data.

Then we modelled the entire PDS (including the QPO bins) with a constant and a constant plus a Lorentzian to account for the QPO feature. While the former yielded a $\chi^{2}$ of 153.6 with 127 dof, adding a Lorentzian improved the $\chi^{2}$ by 23.9, i.e., resulted in a $\chi^{2}$ of 129.8 with 124 dof. This measurement forms the basis for our Monte Carlo simulations described in the following sections. The best-fit QPO centroid is $224.4 \pm 1.0 \mathrm{~Hz}$ respectively.

The ratio of the sum of all the power values within the QPO width to error on that sum gives a quick estimate of the signal-to-noise of the QPO. For the $225 \mathrm{~Hz}$ feature this value is $0.0295 / 0.0061=4.8$. Combined with the mean source count rate of $2.62 \pm 0.01$ counts $/ \mathrm{sec}$, these values can also be used to estimate the fractional root-mean-squared (rms) amplitude of the QPO to be $100 \times \sqrt{0.0295 * 8 / 2.62}=30 \pm 3 \%(8 \mathrm{~Hz}$ is the frequency resolution here). 


\subsubsection{Confirming white noise at lower $(\mathrm{mHz})$ frequencies using longer/continuous light curves from XMM-Newton}

To ensure that AT2018cow's soft X-ray PDS is consistent with white noise at lower frequencies, i.e., $10^{-3}-10^{-2} \mathrm{~Hz}$ we used a $\sim 30 \mathrm{ks} X M M$-Newton exposure that coincided with NICER monitoring. NICER data are not ideal for sampling at these low frequencies because of short exposures. We extracted an average $0.25-2.5 \mathrm{keV}$ XMM-Newton PDS to find that there is no evidence for red noise down to in the frequency range of $\sim 10^{-3}$ - a few Hz. The EPIC-pn PDS with a Nyquist frequency of $6.8 \mathrm{~Hz}$ is shown in Fig. S3.

Also, if there is strong red noise at low frequencies (or long timescales) that can manifest as variability on faster timescales. This effect is known as red noise leakage (41). In the present context, this means that if there is strong red noise below $1 / 256 \mathrm{~Hz}$, that can, in principle, leak into the 100s of $\mathrm{Hz}$ range. The PDS in Fig. S3 also shows that the red noise leakage affect is negligible.

Based on all the above described tests and Figs. S1, S2 and S3 we conclude that over the frequency range of $1 / 256$ to $1024 \mathrm{~Hz}$ the effect of red noise is negligible and that the PDS is consistent with being white.

\subsubsection{Monte Carlo Simulations to Estimate Global Statistical Significance}

When the potential signal you are trying to test for is broad and distributed over several frequency bins, the standard approach of estimating significance based on just the highest bin will not suffice. As it will not include the contribution from all the QPO bins it will fail to capture the true significance estimate. Therefore, we devise an approach that can account for multiple frequency bins. The steps are as follows:

1. After establishing that AT2018cow's soft X-ray variability, on timescales of $1 / 256 \mathrm{~Hz}$ to $1024 \mathrm{~Hz}$ is white, i.e., frequency-independent, we generate a set of 105256 -s "simulated" light curves by simply randomly shuffling each observed light curve independently. In practice (in Python) this is done using numpy's random. shuffle function.

2. Then we extracted an "simulated" average PDS from these shuffled light curves and rebinned it to a frequency resolution of $8 \mathrm{~Hz}$ just like the real PDS in Fig. 1.

3. We then searched for a QPO within this simulated average PDS at all frequencies, i.e., we fit a constant and a constant + a Lorentzian model with the centroid constrained to the ends of each frequency bin and the width allowed to be free, and computed an array of $\Delta \chi^{2}$ values.

4. Finally, we record the maximum $\Delta \chi^{2}$ value from the array of $\Delta \chi^{2}$ value from step 3

The above steps were repeated $10^{5}$ times to get an array of $10^{5}$ maximum $\Delta \chi^{2}$ values. We used 8 cores on a laptop for these simulations which took a total of roughly 180 hours for 
$10^{5}$ simulations. From these measurements we computed the probability to exceed a certain $\Delta \chi^{2}$ value, i.e., 1-CDF. This is shown in the right panel of Fig. 1. For guidance, the $99.73 \%$ and $99.99 \%$ confidence levels are indicated. The QPO found in NICER data is statistically significant at roughly $2 \times 10^{-4}$ level which translates to $3.7 \sigma$ equivalent for a normal distribution.

\subsection{Ruling out an instrumental and a non-astrophysical origin}

All NICER events have two types of pulse height amplitude (PHA) data: the "slow" PHA derived from the slow chain electronics and the "fast" PHA (PHA_FAST) derived by the fast chain electronics. The standard NICER calibration scripts convert this information into pulse invariant (PI) and PI_FAST, respectively. For events with energies $\lesssim 600 \mathrm{eV}$ the fast-chain is not triggered and thus PI_FAST is undefined. Although NICERs XTI is a non-imaging instrument, there is a way to separate the background (particles, cosmic X-rays, and low-energy/optical light loading events) and the source events using the values of PI and PI_FAST. The X-ray events from an on-axis astrophysical source, high-energy particles, light loading, and the cosmic X-ray background occupy a separate region of the PI vs PI_RATIO (PI/PIFAST) plot $^{\S}$.

To ensure the detected signal does not originate from any of these three types of background, we extracted an average PDS of all three backgrounds using their corresponding events within the GTIs. There was no evidence of a variability enhancement around $225 \mathrm{~Hz}$ (or elsewhere) in any of these three backgrounds (see Fig. S4). We describe each of these analyses in a bit more detail below.

\subsubsection{The signal is not present in the particle background}

High energy particles from space can interact with the silicon material in NICER/XTIs SDDs to produce charge and mimic X-ray events. These particle events are registered as enhancements in the so-called overshoot rate for each FPM. Also, because particles have energies much higher than NICERs nominal bandpass of $0.25-12 \mathrm{keV}$ their incidence is also evident in the $13-15 \mathrm{keV}$ count rate. Thus, if this QPO signal were from background particle events it would also be present in the overshoot and the $13-15 \mathrm{keV}$ data.

We extracted the overshoot only event lists using the NICER data reduction pipeline described above but with a modified event flag EVENT_FLAG=bxxx01x. This particular choice selects only the overshoot events. Then, similar to our analysis on source events, we applied barycenter correction on these unfiltered (but calibrated) events. Because overshoots do not contain a PI value they cannot be energy calibrated like real X-ray events. We then extracted the average PDS using only the events within the standard GTIs (see the top-left panel of Fig. S4). There is no evidence for an excess variability anywhere in the PDS.

High-energy events with energies in between 13 and $15 \mathrm{keV}$ that includes the trumpet and all PI_RATIOs were extracted using the standard NICER tools. Then they were barycentercorrected using the barycorr tool similar to the source events. The average PDS from these

\footnotetext{
$\S_{\text {https://heasarc.gsfc.nasa.gov/docs/nicer/mission_guide/ }}$
} 
events is shown in the top-right panel of Fig. S4 and does not show any obvious QPO like features anywhere in the considered frequency range.

The average count rate of the overshoots and the $13-17 \mathrm{keV}$ were 18.7 and 0.18 counts/sec, respectively. The former value is much higher than AT2018cow's mean soft X-ray count rate of 2.62 counts/s. Therefore, if the $225 \mathrm{~Hz}$ QPO were from background particles it would have shown up in the top-left panel of Fig. S4 (sensitivity towards a QPO increases linearly with count rate (40)). Thus, the top two panels of Fig. S4 allows us to rule out a particle background origin for the $225 \mathrm{~Hz}$ QPO.

\subsubsection{The signal is not due to optical light leak events}

Optical light, either directly from the Sun or bright Earth at low angles, or from reflections off ISS surfaces can make its way into the FPMs. This manifests as a noise peak in each FPM whose energy spectrum peaks at an energy below $0.25 \mathrm{keV}$. However, when this light loading, which is time variable, is strong, the tail end of the noise distribution can leak into higher energies $(>0.25$ $\mathrm{keV}$ ) and contaminate the source events. This effect is especially important for faint targets like AT2018cow. The event filtering described in section 1.1 should already remove epochs of high light loading. To further ensure that the QPO signal is not a result of variability of "light leak" events, if any, we extracted a 0.0-0.2 keV PDS. Similar to the inband PDS we first extracted the $0.0-0.2 \mathrm{keV}$ events using the standard NICER tasks. We then barycenter-corrected them and then computed an average PDS using events within the standard GTIs. The resulting power spectrum shown in the bottom-left panel of Fig. S4 allows us to rule out a light leak origin for this QPO.

\subsubsection{The signal is not present in cosmic $\mathrm{X}$-ray background events within NICER's FoV}

Although NICER/XTI is a non-imaging instrument, it's design with the slow and the fast chain electronics allows us to separate on-axis events from the off-axis ones. While the on-axis events make a "Trumpet"-like cluster in the PI vs PI_RATIO plot, the off-axis events, i.e., those from cosmic X-rays and other point sources in the FoV, fall above the "Trumpet". If the QPO signal is associated with AT2018cow (placed on-axis during each observation) then it should not be present in the trumpet-rejected events. The inband PDS shown in Fig. 1 already excludes trumpet-reject events. However, to be absolutely sure, we extract an average PDS of the trumpet-rejected events within the standard GTIs. We extracted the trumpet-rejected events by first extracting the standard cleaned event lists with $t r u m p f i l t=\mathrm{NO}$. These were barycentercorrected and then screened to only include events between 0.25 and $10 \mathrm{keV}$ that fell above the trumpet edge defined by the curve PI RATIO $=1.1+120 /$ PI. An average PDS was extracted in same manner as the inband PDS. This is shown in the bottom-right panel of Fig. S4 and it is evident that a $225 \mathrm{~Hz}$ QPO is not present in events that describe the cosmic X-ray background and any contaminating sources in the field of view. 


\subsubsection{The signal is present in all Measurement and Power Units (MPUs)}

If this signal is astrophysical in origin then it must be uniformly distributed across all the 52

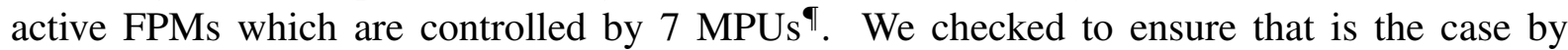
extracting several average PDS, but with data from one MPU removed at a time. The resulting 7 PDS with the ID of the removed MPU at the top are shown in Fig. S5. The QPO is evident in all the 7 PDS with a fractional rms amplitude value consistent with each other. This demonstrates that the QPO events are uniformly distributed across all MPUs and not just limited to any single unit.

\subsubsection{The signal is not due to "GPS" noise}

One other plausible instrument-related source of origin of this signal is the co-called "Global Position System (GPS) noise. During the ground testing of NICERs MPUs anomalous cross-talk signals between the FPM input line and the GPS pulse-per-second (PPS) line were observed. This can result in certain FPMs registering pseudo events (non-cosmic) immediately following a GPS PPS tick, usually within the first $4 \mathrm{~ms}$ after the tick. In principle, this could produce a 1 second QPO with roughly a $4 \mathrm{~ms}$ QPO width, although it has never been reported in any of the analysis of several NICER targets thus far. This is obviously at a different frequency compared to the $225 \mathrm{~Hz}$ QPO from observations of AT2018cow. Nevertheless, we rule out GPS noise as the source of AT2018cows QPO by first removing all events with time stamps occurring within $10 \mathrm{~ms}$ of an integer second of the original non-barycenter corrected data. Then, we barycenter corrected the remaining events and extracted an average PDS in the exact same manner as the PDS in Fig. 1. The resulting PDS shown in Fig. S6 is virtually indistinguishable from the left panel of Fig. 1. This is not too surprising as the above only excludes a small fraction of all events. Fig. S6 shows that the signal is still present after removing the plausible GPS noise events and rules out GPS noise as the origin of this QPO.

\subsubsection{AT2018cow dominates the $\mathbf{X}$-ray emission in NICER/XTI's FoV}

NICER/XTI is a non-imaging detector with a field of view of roughly $30 \operatorname{arcmin}^{2}$. Thus it is plausible that a contaminating point source other than AT2018cow could have produced this QPO signal. To investigate this possibility, we extracted an image by stacking the entire Neil Gehrels Swift/XRT archival data of AT2018cow (see the right panel of Fig. 3). It is clear from these images that AT2018cow is the brightest point source within XTI's FoV. The contribution from the other point sources is negligible.

Furthermore, we can directly compare NICER/XTI light curve of AT2018cow's FoV with resolved XRT light curve. The variability features in XRT light curve are also evident in XTI data (see Fig. 2). This independently implies that AT2018cow dominates the X-ray emission in XTI's FoV.

Thttps://heasarc.gsfc.nasa.gov/docs/nicer/mission_guide/ 
As a final check, we also investigated a late time XMM-Newton image of AT2018cow's FoV to rule out contamination by a point source within XRT or EPIC instrument's point spread functions. The left panel of Fig. 3 show MOS1 image of AT2018cow's FoV at late times. We used MOS1 because it offers the best pixel size of 4.1". It is evident that long after AT2018cow's optical decay, there is no X-ray emission present at its location and rules out a contaminating source very close to the position of AT2018cow.

The combination of the above three analyses affirms that the majority of emission detected by XTI between MJD 58290.87 and 58349.11 and thus the $225 \mathrm{~Hz}$ signal originates from AT2018cow.

\subsubsection{A similar signal is NOT present in any AGN monitored by NICER during the same epoch}

AGN host supermassive black holes $\left(\gtrsim 10^{5} \mathrm{M}_{\odot}\right)$ and hence causality argument suggests that they should not show any variability on timescales of milliseconds, i.e., hundreds of Hz. Therefore, to be absolutely sure that the $225 \mathrm{~Hz}$ QPO signal is unique to AT2018cow data we also extracted average PDS of 3 active galactic nuclei that NICER monitored during the same epoch, i.e., only using their observations between MJD 58290.87 and 58349.11. These resulting average PDS are shown in Fig. S8 and a $225 \mathrm{~Hz}$ feature is not seen in any of them.

\subsection{QPO is highly persistent}

The fact that the QPO is detected in the average PDS extracted from data accumulated over a temporal baseline of $\approx 60$ days suggests that the QPO is likely present for a significant fraction of the X-ray monitoring. To further test this we estimated the strength (signal-to-noise ratio) of the QPO as a function of the accumulated exposure. We started with the first $5 \mathrm{ks}$ of exposure, extracted an average PDS, and fit the $225 \mathrm{~Hz}$ feature with a Lorentzian. From thereon, for every additional 256-s exposure, we repeated this process of extracting an average PDS, followed by modelling the $225 \mathrm{~Hz}$ feature with a Lorentzian. At every point, the QPO strength was calculated as the ratio of the sum of the powers within the width of the best-fit QPO and its errorbar. A normalized QPO strength was also estimated by dividing the QPO strength with the average count rate which itself evolves with the accumulated exposure. It is evident from Fig. S7 that the overall strength of the QPO gradually increases with increasing exposure. This demonstrates that:

1. The QPO was persistent for a substantial fraction of the $60 \mathrm{~d}$ monitoring program, i.e., for several hundreds of millions of cycles ( $\sim 60$ days/4.4ms).

2. The QPO signal does not originate from any single exposure of the NICER monitoring.

The same result can be visualized in the form of a movie showing the evolution of the average PDS as a function of increasing exposure. This can be found as a supplementary file (Movie S1). A gif version is also available. 


\section{Implications for a magnetar scenario}

The bolometric light curve of AT2018cow reached a luminosity of $\sim 4 \times 10^{44} \mathrm{erg} \mathrm{s}^{-1}$ on time a timescale of a few days after the explosion, and the luminosity decayed as $L_{\mathrm{e}} \propto t^{-\alpha}$, where $\alpha$ $\approx 2.5$ at times, $\mathrm{t} \gtrsim \mathrm{t}_{e} \sim 10^{3}-10^{5} \mathrm{~s}$. The central engine responsible for powering the optical and X-ray emission must supply a total energy $\mathrm{E}_{\mathrm{e}} \sim 10^{50}-10^{52}$ erg over a characteristic timescale of $t_{e}$. Degeneracy exists in these properties because we do not know how much of the kinetic energy of the ejecta is supplied by the initial explosion itself, versus injected at later times by the engine.

Two models for the central engine include: (1) a stellar-mass black hole of mass $\sim 10-30 \mathrm{M}_{\odot}$ created by the failed explosion of a very massive star, which is accreting fall-back material from the outer layers of the extended progenitor at a highly super-Eddington rate; (2) a magnetar with a rapid birth period $\mathrm{P}_{0} \sim \mathrm{ms}$ and a strong dipole magnetic field $\mathrm{B} \sim 10^{14}-10^{15} \mathrm{G}$. The magnetar may be spinning down in isolation, or it may be accreting fall-back material as in the black hole. The latter scenario may be supported by the predict decline rate of the engine luminosity $L_{\mathrm{e}} \propto t^{-2.38}$.

We now consider models for the origin of the QPO-like feature at $\mathrm{f}_{\mathrm{QPO}}=225 \mathrm{~Hz}$, assuming it is related to the spin period of a magnetar, $\mathrm{P}_{\mathrm{QPO}}=1 / \mathrm{f}_{\mathrm{QPO}} \approx 4.44 \mathrm{~ms}$ or half of that value $\left(\mathrm{P}_{\mathrm{QPO}}=8.9 \mathrm{~ms}\right)$. Alternatively, the $\mathrm{QPO}$ could arise directly in the accretion disk (of the black hole or neutron star), e.g. as in an X-ray binary QPO, a possibility which is not addressed below.

\subsection{Spin of an isolated pulsar or magnetar}

Here we consider the possibility that the observed QPO at $225 \mathrm{~Hz}$ is the spin frequency of a pulsar or magnetar. The traditional picture of pulsar spin evolution (see, e.g., (42), for a review) is that the pulsar's rotational energy

$$
E_{\mathrm{rot}}(\nu)=\frac{I}{2}(2 \pi \nu)^{2} \sim 1 \times 10^{51} \operatorname{erg}\left(\frac{\nu}{225 \mathrm{~Hz}}\right)^{2},
$$

where $I \sim 10^{45} \mathrm{~g} \mathrm{~cm}^{2}$ is the stellar moment of inertia, can supply energy at a rate

$$
L_{\mathrm{sd}}=4 \pi^{2} I \nu \dot{\nu} \sim 3 \times 10^{43} \operatorname{erg~s}^{-1}\left(\frac{\nu}{225 \mathrm{~Hz}}\right)\left(\frac{\dot{\nu}}{3 \times 10^{-6} \mathrm{~Hz} \mathrm{~s}^{-1}}\right)
$$

to power radiation from a rotating magnetic dipole at a rate $L_{\operatorname{mag}} \propto B^{2} \nu^{4}$. Equating the two rates yields an estimate of the pulsar magnetic field

$$
B \approx 2 \times 10^{13} \mathrm{G}\left(\frac{\nu}{225 \mathrm{~Hz}}\right)^{-3 / 2}\left(\frac{-\dot{\nu}}{3 \times 10^{-6} \mathrm{~Hz} \mathrm{~s}^{-1}}\right)^{1 / 2}
$$


and evolution of spin frequency

$$
\nu=\nu_{0}\left(1+t / t_{\mathrm{sd}}\right)^{-1 / 2},
$$

which then leads to

$$
L_{\mathrm{sd}}=\frac{E_{\mathrm{rot}}\left(\nu_{0}\right)}{t_{\mathrm{sd}}} \frac{1}{\left(1+t / t_{\mathrm{sd}}\right)^{2}}=\frac{3 \times 10^{43} \mathrm{erg} \mathrm{s}^{-1}}{\left(1+t / t_{\mathrm{sd}}\right)^{2}}\left(\frac{\nu_{0}}{225 \mathrm{~Hz}}\right)\left(\frac{-\dot{\nu}_{0}}{3 \times 10^{-6} \mathrm{~Hz} \mathrm{~s}^{-1}}\right),
$$

where the characteristic timescale for spin-down is

$t_{\mathrm{sd}}=-\frac{\nu_{0}}{2 \dot{\nu}_{0}} \approx 430 \mathrm{~d}\left(\frac{\nu_{0}}{225 \mathrm{~Hz}}\right)\left(\frac{-\dot{\nu}_{0}}{3 \times 10^{-6} \mathrm{~Hz} \mathrm{~s}^{-1}}\right)^{-1}=430 \mathrm{~d}\left(\frac{\nu_{0}}{225 \mathrm{~Hz}}\right)^{-2}\left(\frac{B}{2 \times 10^{13} \mathrm{G}}\right)^{-2}$

and $\nu_{0}$ and $\dot{\nu}_{0}$ are the initial spin frequency and spin frequency time derivative, respectively. One can see from equation (S5) that the luminosity is constant at times $t \ll t_{\text {sd }}$ and decreases as $L_{\mathrm{sd}} \propto t^{-2}$ when $t \gg t_{\mathrm{sd}}$.

The QPO in AT2018cow is observed to persist at $225 \mathrm{~Hz}$ for about 60 days with a width of $<16 \mathrm{~Hz}$. This constrains the frequency change to

$$
|\dot{\nu}|<16 \mathrm{~Hz} / 60 \mathrm{~d}=3 \times 10^{-6} \mathrm{~Hz} \mathrm{~s}^{-1}
$$

and magnetic field and spin-down timescale to $B<2 \times 10^{13} \mathrm{G}$ and $t_{\mathrm{sd}}>430 \mathrm{~d}$, respectively. Even stricter constraints of

$$
-\dot{\nu}<3 \times 10^{-8} \mathrm{~Hz} \mathrm{~s}^{-1}\left(\frac{L}{3 \times 10^{41} \mathrm{erg} \mathrm{s}^{-1}}\right)
$$

and $B<2 \times 10^{12} \mathrm{G}\left(L / 3 \times 10^{41} \mathrm{erg} \mathrm{s}^{-1}\right)^{1 / 2}$ are obtained from equations (S3) and (S5) by noting that the bolometric and X-ray luminosities are seen to decrease at times $<60 \mathrm{~d}$ to a lowest value of $\sim 10^{41} \mathrm{erg} \mathrm{s}^{-1}(5)$.

The above discussion rules out the possibility of a millisecond magnetar powering AT2018cow. If $B>10^{14} \mathrm{G}$, then $t_{\mathrm{sd}}<20 \mathrm{~d}$ and $-\dot{\nu}>1 \times 10^{-4} \mathrm{~Hz} \mathrm{~s}^{-1}$. The latter in particular implies the QPO frequency would have decreased by more than $100 \mathrm{~Hz}$ in a span of just 10 days.

\subsection{Neutron star accretion}

Alternatively, a remnant magnetar may be accreting fall-back matter from its birthing supernova explosion, and the observed engine activity is either accretion or spin-down powered. The rate of mass fall-back can be written (e.g., (43))

$$
\dot{M}(t)=\frac{2}{3} \frac{M_{\mathrm{acc}}}{t_{\mathrm{acc}}} \frac{1}{\left(1+t / t_{\mathrm{acc}}\right)^{5 / 3}},
$$


where $M_{\text {acc }}$ is the total quantity of returning mass and $t_{\text {acc }} \sim t_{\mathrm{ff}} \sim(G \bar{\rho})^{-1 / 2}$ is the characteristic fall-back time which depends on the mean density $\bar{\rho}$ of the layer of progenitor star contributing to $M_{\text {acc }}$. For an extended star with hydrogen-rich ejecta like a blue supergiant $\left(\bar{\rho} \sim 10^{-5}-10^{-3}\right.$ $\mathrm{g} \mathrm{cm}^{-3}$ ), we have $t_{\mathrm{acc}} \sim 10^{5}-10^{6} \mathrm{~s}$, compatible with the timescale of engine activity for AT2018cow (5). A neutron star of initial mass $M_{\mathrm{ns}} \approx 1.4 M_{\odot}$ can accrete at most $M_{\mathrm{acc}} \approx$ $0.8 M_{\odot}$ before collapsing into a black hole and thus indicates a maximum accretion rate in the range $\lesssim 10^{-6}-10^{-5} M_{\odot} \mathrm{s}^{-1}$, i.e., 9-10 orders of magnitude above the Eddington accretion rate for a solar-mass compact object. By comparison, to explain the peak engine luminosity of AT2018cow $\left(\sim 10^{45} \mathrm{erg} \mathrm{s}^{-1}\right.$ or 7 orders of magnitude above the Eddington luminosity) through accretion would require either a lower peak accretion rate $\dot{M} \sim 10^{-8} M_{\odot} \mathrm{s}^{-1}$ or inefficient production of X-rays by the accretion flow. On timescales of several weeks, relevant to the epoch of the observed QPO, the accretion rate is $\sim 2$ orders of magnitude lower than at peak, i.e. in the characteristic range

$$
\dot{M}\left(t_{\mathrm{QPO}}\right) \sim 10^{-10}-10^{-8} M_{\odot} \mathrm{s}^{-1}
$$

The Alfvén radius of the accretion flow, at which the ram pressure of the incoming flow is balance by magnetic forces, is given by (e.g., (43))

$$
R_{A} \approx 40 B_{12}^{4 / 7} \dot{M}_{-9}^{-2 / 7} M_{1.4}^{-1 / 7} \mathrm{~km}
$$

where $\dot{M}_{-9}=\dot{M} /\left(10^{-9} \mathrm{M}_{\odot} \mathrm{s}^{-1}\right)$ and now we have normalized the surface magnetic field $\mathrm{B}_{12}=$ $\mathrm{B} / 10^{12} \mathrm{G}$ to a lower value more akin to a radio pulsar than a magnetar. If the magnetic field is sufficiently large that $R_{\mathrm{A}}$ exceeds the neutron star radius $\approx 12 \mathrm{~km}$, i.e. if

$$
B_{12} \gtrsim 0.1 \dot{M}_{-9}^{1 / 2} M_{1.4}^{1 / 4}
$$

then the accretion flow will be directed from the disk onto the magnetic axis of the neutron star, providing a possible mechanism to induce periodicity in the signal on the rotation period.

In order for accretion to occur, a neutron star must rotate slow enough that it is not in an ejector phase (44-46), and this occurs approximately when the Alfvén radius $R_{\mathrm{A}}$ is smaller than the light cylinder radius $R_{\mathrm{lc}} \equiv c / 2 \pi \nu$. This then implies

$$
B_{12}<20 \dot{M}_{-9}^{1 / 2} M_{1.4}^{1 / 4}\left(\frac{\nu}{225 \mathrm{~Hz}}\right)^{-7 / 4}
$$

Accretion also affects the spin-down rate of the neutron star, driving the spin-period to an 
equilibrium value given by $(43,45,47,48)^{\|}$

$$
P_{\mathrm{eq}} \approx 4 B_{12}^{6 / 7} \dot{M}_{-9}^{-3 / 7} M_{1.4}^{-5 / 7} \mathrm{~ms}
$$

Explaining the observed $\mathrm{QPO}\left(\mathrm{P}_{\mathrm{eq}} \approx 1-2 P_{\mathrm{QPO}} \approx 4.4-8.8 \mathrm{~ms}\right)$ thus requires an accretion rate of

$$
\dot{M}\left(t_{\mathrm{QPO}}\right) \approx(2-9) \times 10^{-10} B_{12}^{2} M_{1.4}^{-5 / 3} M_{\odot} \mathrm{s}^{-1},
$$

consistent with our estimate in eq. S10. However the timescale to reach this equilibrium (Ref. (43), their eq. 21 ; see also $(45-47,50)$ ),

$$
\tau_{e q} \approx 400 \mathrm{~d} B_{12}^{-8 / 7} \dot{M}_{-9}^{-3 / 7} M_{1.4}^{16 / 7} \underset{\dot{M}=\tilde{M}_{\mathrm{QPO}}}{\approx}(400-900) B_{12}^{-2} M_{1.4}^{16 / 7} \mathrm{~d}
$$

can be long. This indicates that $P \approx P_{\mathrm{eq}}$ will only be achieved on timescales of the observed QPO $\left(\tau_{e q} \ll 25 \mathrm{~d}\right.$ ) if $B$ (or, equivalently, $\left.\dot{M} \propto B^{2}\right)$ is sufficiently high, $B_{12} \gtrsim 3$. In such a case that $\tau_{\mathrm{eq}} \gg t_{\mathrm{QPO}}$, then the spin period would reflect that of the neutron star at birth rather than $P_{e q}$, the equilibrium value achieved through accretion.

In this scenario, if $P=P_{\text {eq }}$ is achieved, then the rotation period of the neutron star (and thus the QPO frequency) should increase in time as (eq. S14)

$$
P_{e q} \propto \dot{M}^{-3 / 7} \underset{\dot{M} \propto t^{-5 / 3}}{\propto} t^{5 / 7},
$$

somewhat faster than in the isolated magnetar case (eq. S4).

One appealing aspect of this scenario is that, again if the equilibrium $P=P_{\mathrm{eq}}$ is achieved, then the luminosity of the neutron star wind is given by (Ref. (43), their eq. 22)

$$
L_{\mathrm{sd}} \approx 1 \times 10^{43} B_{12}^{-6 / 7} \dot{M}_{-9}^{10 / 7} M_{1.4}^{12 / 7} \operatorname{erg~s}^{-1} \underset{\dot{M}=\dot{M}\left(t_{\mathrm{QPO}}\right)}{\approx}\left(10^{42}-10^{43}\right) B_{12}^{2} \mathrm{erg} \mathrm{s}^{-1},
$$

similar to the luminosity of AT2018cow on timescales of a few weeks. Furthermore, if $\dot{M} \propto$ $t^{-5 / 3}$ then one predicts a decay in the luminosity $L_{\text {sd }} \propto \dot{M}^{10 / 7} \propto t^{-2.38}$, close to the observed decay of the bolometric light curve of AT2018cow (e.g., (5)).

This is comparable to the accretion power onto the neutron star surface,

$$
L_{\mathrm{acc}} \approx \frac{G M_{\mathrm{ns}} \dot{M}}{R_{\mathrm{ns}}} \approx 3 \times 10^{44} \dot{M}_{-9} M_{1.4} \mathrm{erg} \mathrm{s}^{-1} \underset{\dot{M}=\dot{M}_{\mathrm{QPO}}}{\approx} 3 \times 10^{42}-2 \times 10^{43} B_{12}^{2} \mathrm{erg} \mathrm{s}^{-1},
$$

which could therefore also contribute to the luminosity. However, given the generally low radiative efficiency of highly super-Eddington accretion flows, its contribution could be swamped

"Unlike in some past work, we assume that this equilibrium is determined by the balance of accretion spin-up and spin-down from the magnetized wind (the latter enhanced as a result of the larger magnetosphere opened by the fall-back (49)). 
by energy injected from the neutron star wind, $L_{\mathrm{sd}}$.

Even with the narrow range of $200-250 \mathrm{~Hz}$ the search space for the pulsar (frequency, start time of the explosion) is rather large. We defer the search for the pulsar to a future work as it requires significant computational resources on a computer cluster unavailable to the authors at present. But we provide the barycenter corrected events as supplementary files so that any reader with access to such a cluster may use it. A sample Python code to load all the events is also provided (load_events.py).

\section{Pulsation search assuming a binary}

To search for possible orbital periodicities, we used the open source PRESTO software** to perform an acceleration search over the frequency-frequency derivative plane. The acceleration search scheme assumes that the compact objects acceleration is roughly constant throughout the observation of duration $T \lesssim P_{\text {orb }} / 10(51)$. We ran the search assuming that any possible signal would drift across a maximum of 100 Fourier frequency bins (zmax of 100). Informed by the centroid frequency and width of the QPO (assumed to be the fundamental frequency) reported above, we narrowed the search frequency window to be over $200-260 \mathrm{~Hz}^{\dagger \dagger}$. The acceleration search yielded no candidates above 3 sigma (single trial probability). Subsequently, we accounted for the possibility of linearly changing accelerations, and carried out a jerk search (52), with PRESTO ${ }^{\ddagger}$. This opens the way to detect other more exotic systems like very compact, relativistic binary systems, and allows searches over longer observations, unlike in acceleration searches. The jerk search would also allow us to recover any lost signal from residual Doppler smearing in the previous acceleration searches (53). In the end, the jerk search also yielded no candidates above 3 sigma (single trial probability).

As described in section 3, the spin of the potential neutron star will evolve in a manner that depends strongly on the actual underlying physical scenario. Exploring all these models is beyond the scope of this work.

${ }^{* *}$ https://github.com/scottransom/presto

${ }^{\dagger} \dagger$ in PRESTO parlance, this is flo of 200, and fhi of 260

$\ddagger \ddagger$ In PRESTO parlance, this is wmax of 300 


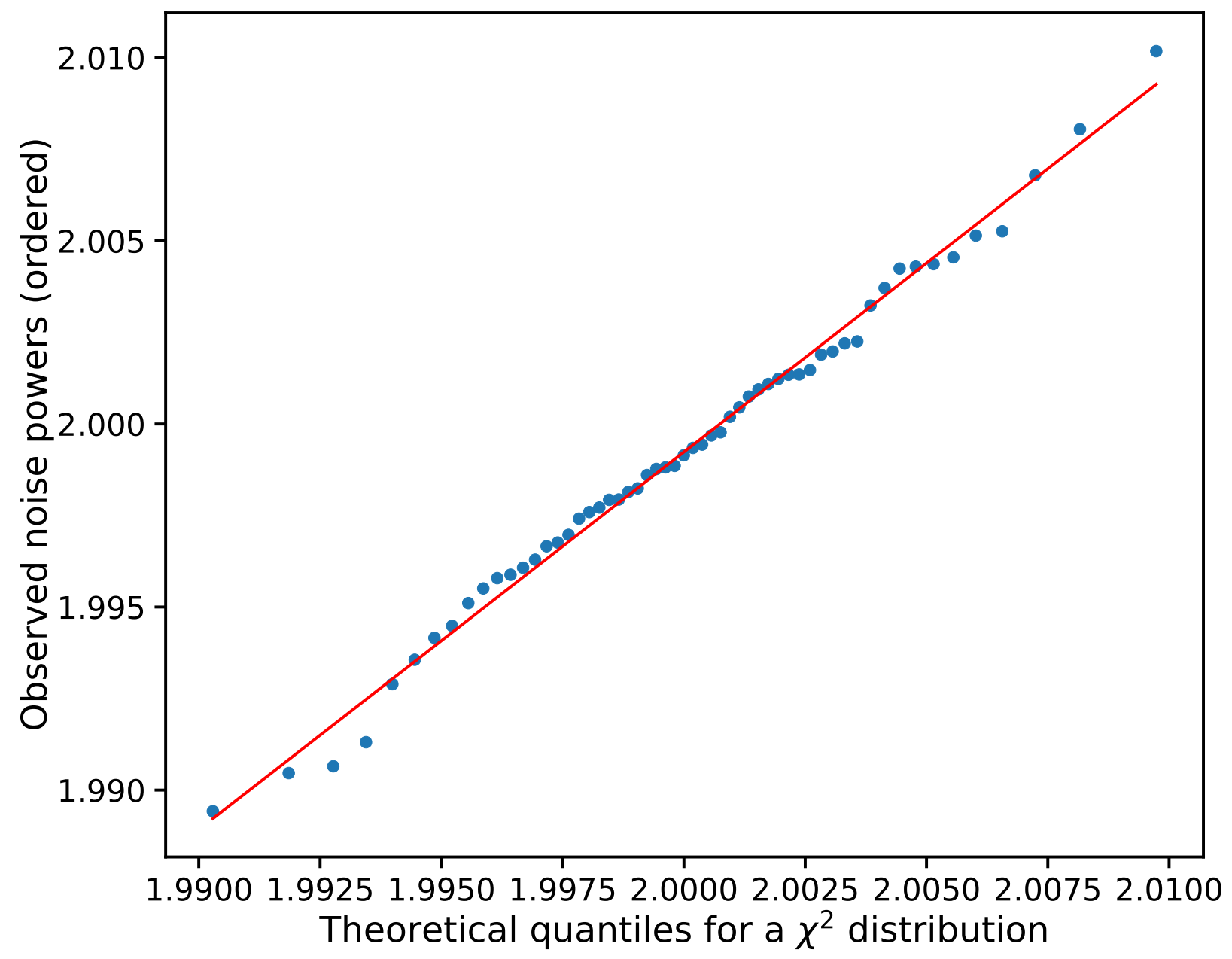

Figure S1: Probability plot to visually assess whether the power values in the PDS continuum are $\chi^{2}$ distributed. This is a qualitative assessment tool that tells us that if the data points lie on a straight line, as they do, they are appear consistent with the theorized model, which in the present case is a $\chi^{2}$ distribution with $2 \times 105 \times 2048$ degrees of freedom (see section 2.2.1 for more details). 
(a) Cumulative Distribution Function of Noise powers
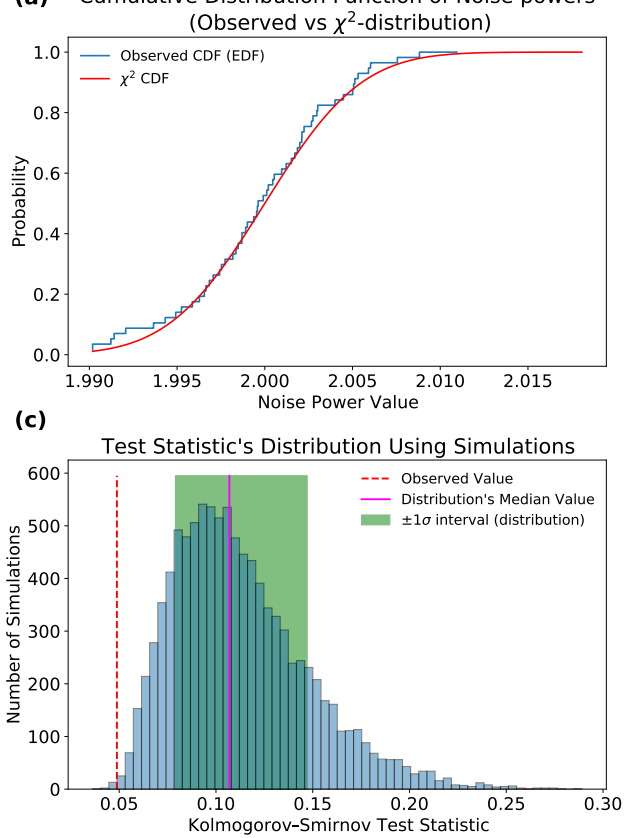
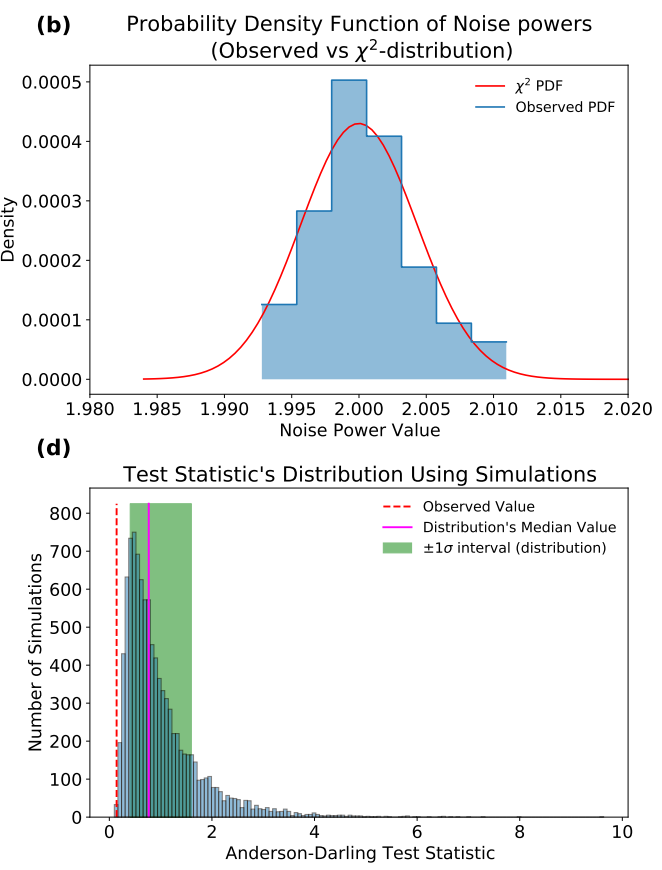

Figure S2: White noise tests for soft X-ray PDS of AT2018cow.Top-left: Comparing the empirical distribution function (EDF) of the values in the PDS continuum (blue histogram) with the cumulative distribution function of a $\chi^{2}$ distribution with $2 \times 105 \times 2048$ degrees of freedom. Top-right: Comparison of the observed probability distribution function (PDF) with the expected PDF of a $\chi^{2}$ distribution with $2 \times 105 \times 2048$ dof. Both the observed PDF and EDF track expeted curves quite well. Bottom-left: The distribution of the K-S statistic derived from EDFs sampled from a $\chi^{2}$ distribution with $2 \times 105 \times 2048$ dof. Bottom-right: Same as bottomleft but using an Anderson-Darling test statistic. Both the test statistic values are consistent with a $\chi^{2}$ distribution and suggest that the PDS continuum is white (see section 2.2.2 for more details). 


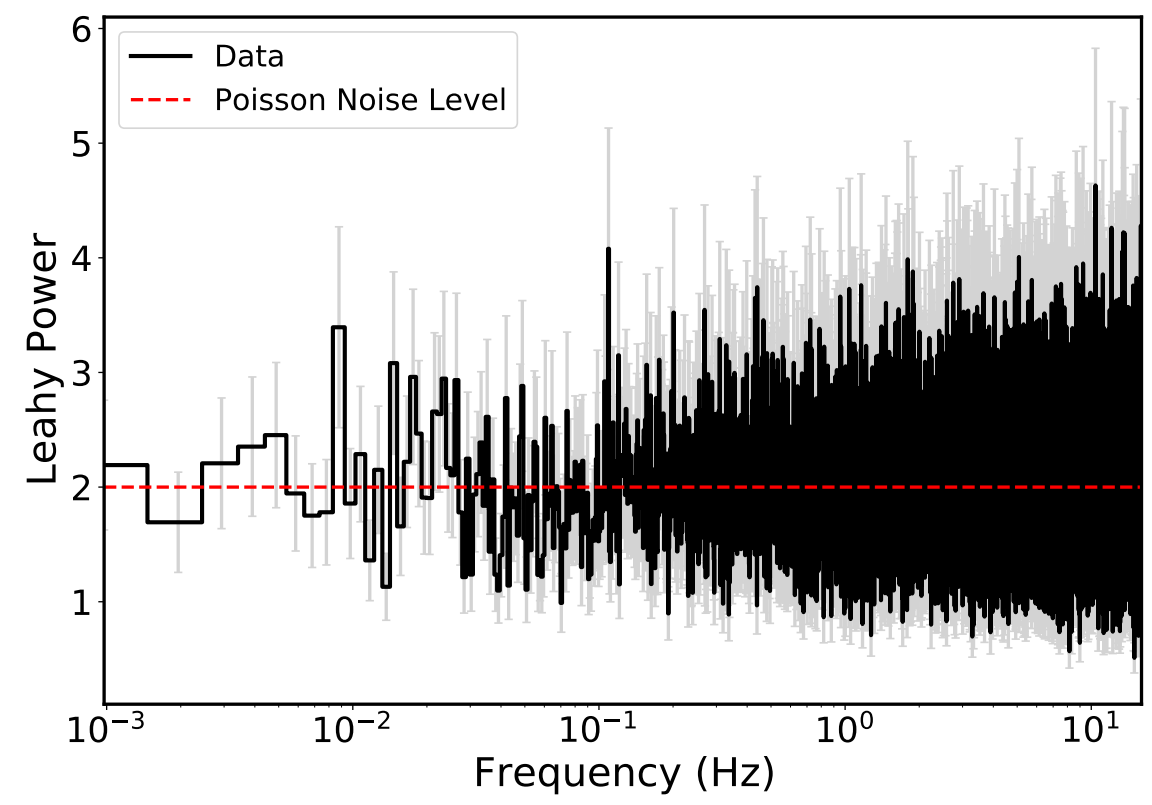

Figure S3: XMM-Newton/EPIC-pn soft X-ray (0.25-2.5 keV) PDS of AT2018cow to assess noise continuum at low frequencies. The PDS was derived by averaging $151024 \mathrm{~s} \mathrm{light}$ curve segments sampled at $1 / 16 \mathrm{~Hz}$. The frequency resolution is $1 / 2048 \mathrm{~Hz}$. It is evident that even at frequencies as low as $1 / 2048 \mathrm{~Hz}$ there is no evidence for red noise. The $225 \mathrm{~Hz}$ QPO in NICER data is outside of this band pass, i.e., beyond EPIC-pn's Nyquist frequency. 

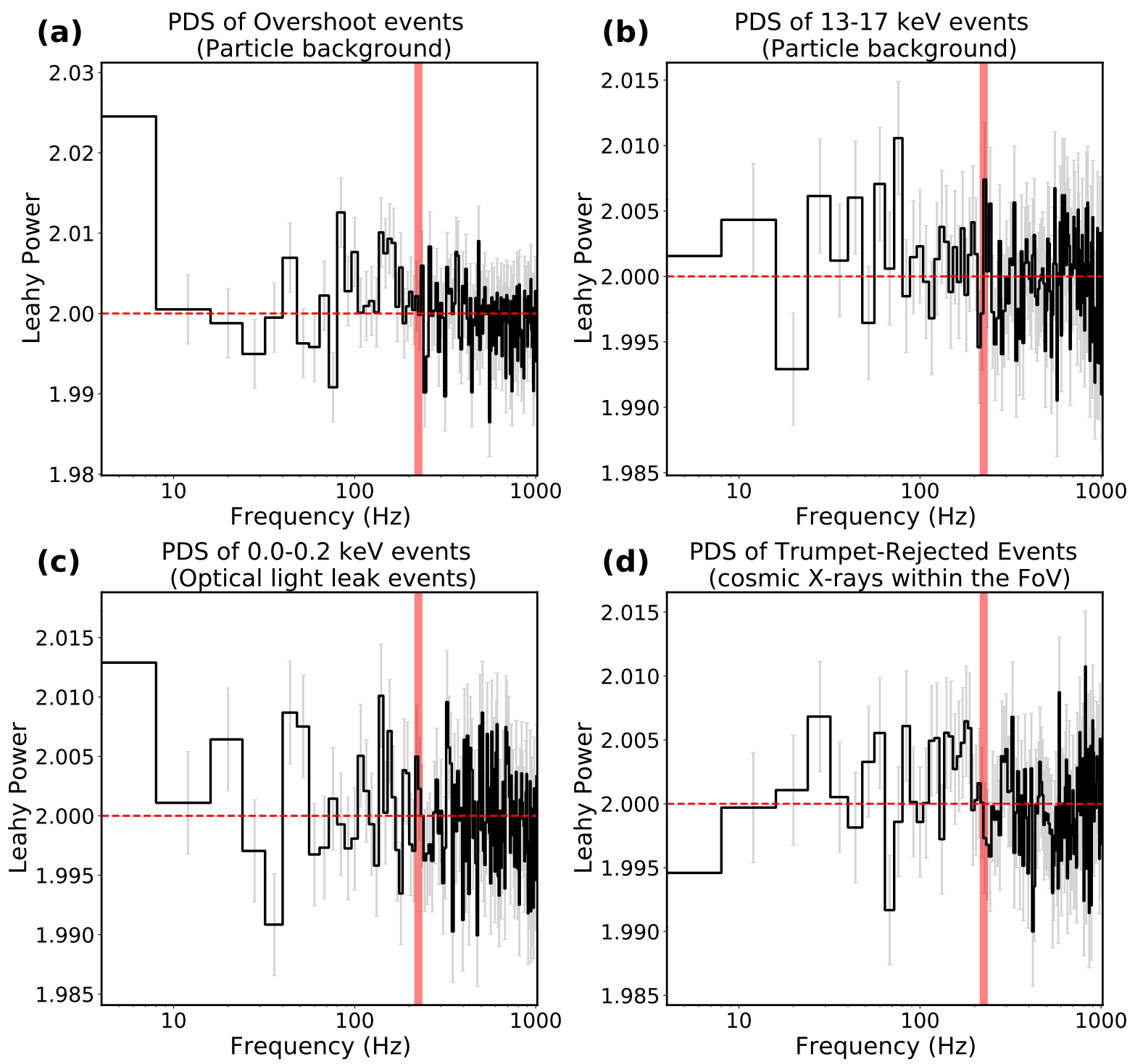

Figure S4: NICER PDS of various types of noise. (a) and (b) show the PDS of the overshoots and the 13-15 keV events, respectively. They both track the particle background. (c) The average PDS of 0.0-0.2 keV events. These are the optical light leak events. (d) The average PDS derived from trumpet-rejected events. These are the off-axis events that track the cosmic $\mathrm{X}$-ray and other sources in the FoV. In all cases, the frequency resolution, the number of spectra averaged and the frequency rebinning is same as the left panel of Fig. 1. The red shaded rectangle shows the location of the $225 \mathrm{~Hz}$ QPO. Clearly, there is no statistically significant evidence for a $225 \mathrm{~Hz}$ QPO in any of these noise power spectra. The dashed horizontal line indicates the expected Poisson noise level of 2. These plots suggest that the $225 \mathrm{~Hz}$ QPO in Fig. 1 does not originate from any of these background events. 

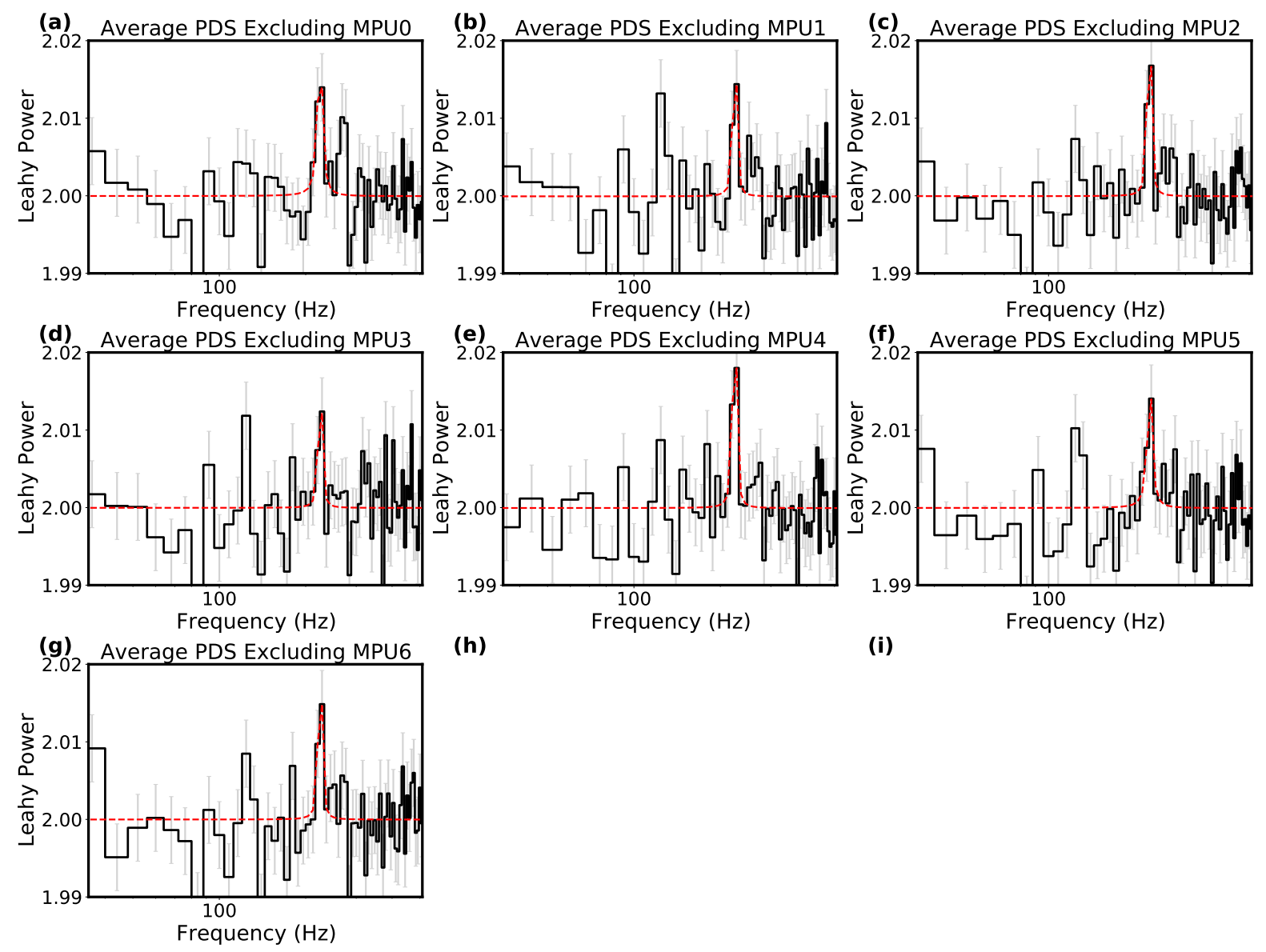

(h)

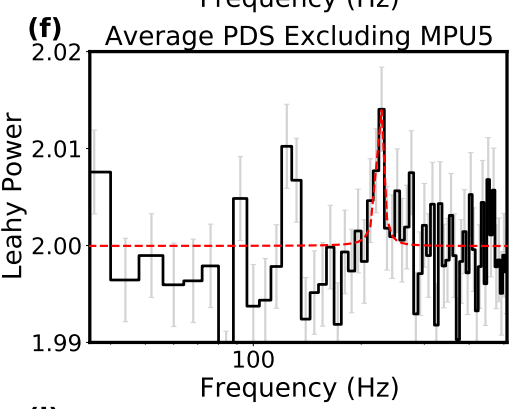

(i)

Figure S5: NICER power spectra with one MPU removed at a time. The QPO properties, i.e., fractional rms strength, centroid and width are all same (within errorbars) across all the above PDS. This demonstrates that the QPO signal is uniformly distributed across all the MPUs and points towards an non-instrumental origin. 


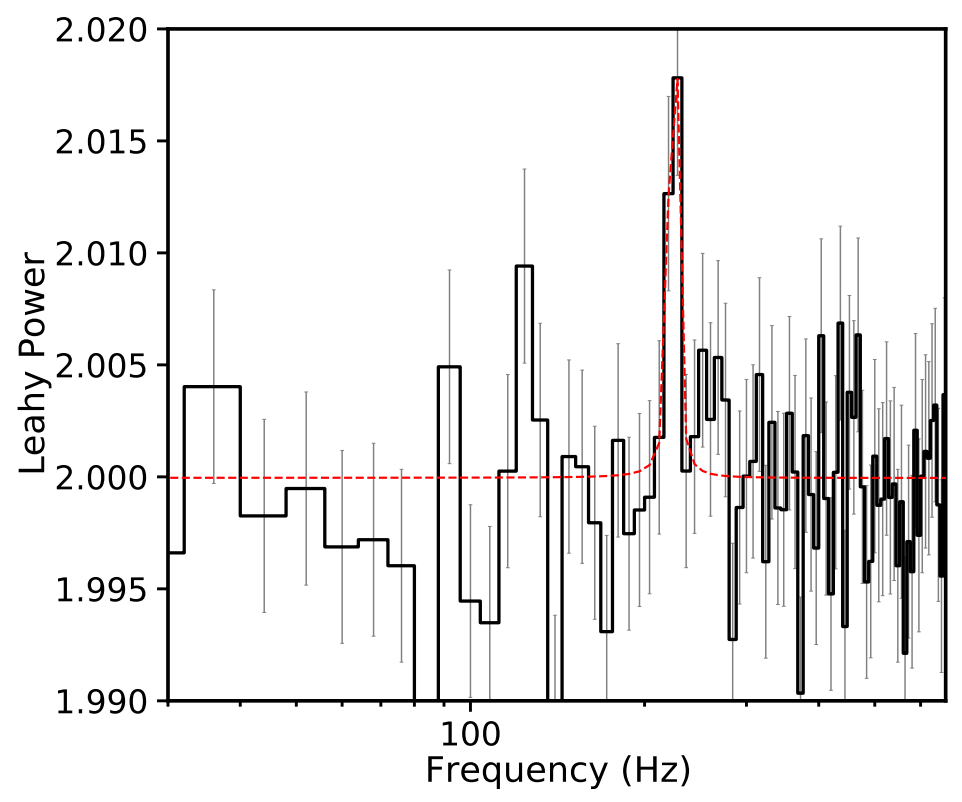

Figure S6: Same power spectra as the left panel of Fig. 1 but with plausible GPS noise events removed. This PDS is indistinguishable from Fig. 1 and demonstrates that the GPS noise, if any, is not the origin of the $225 \mathrm{~Hz}$ QPO (see section 2.3.5 for more details) 


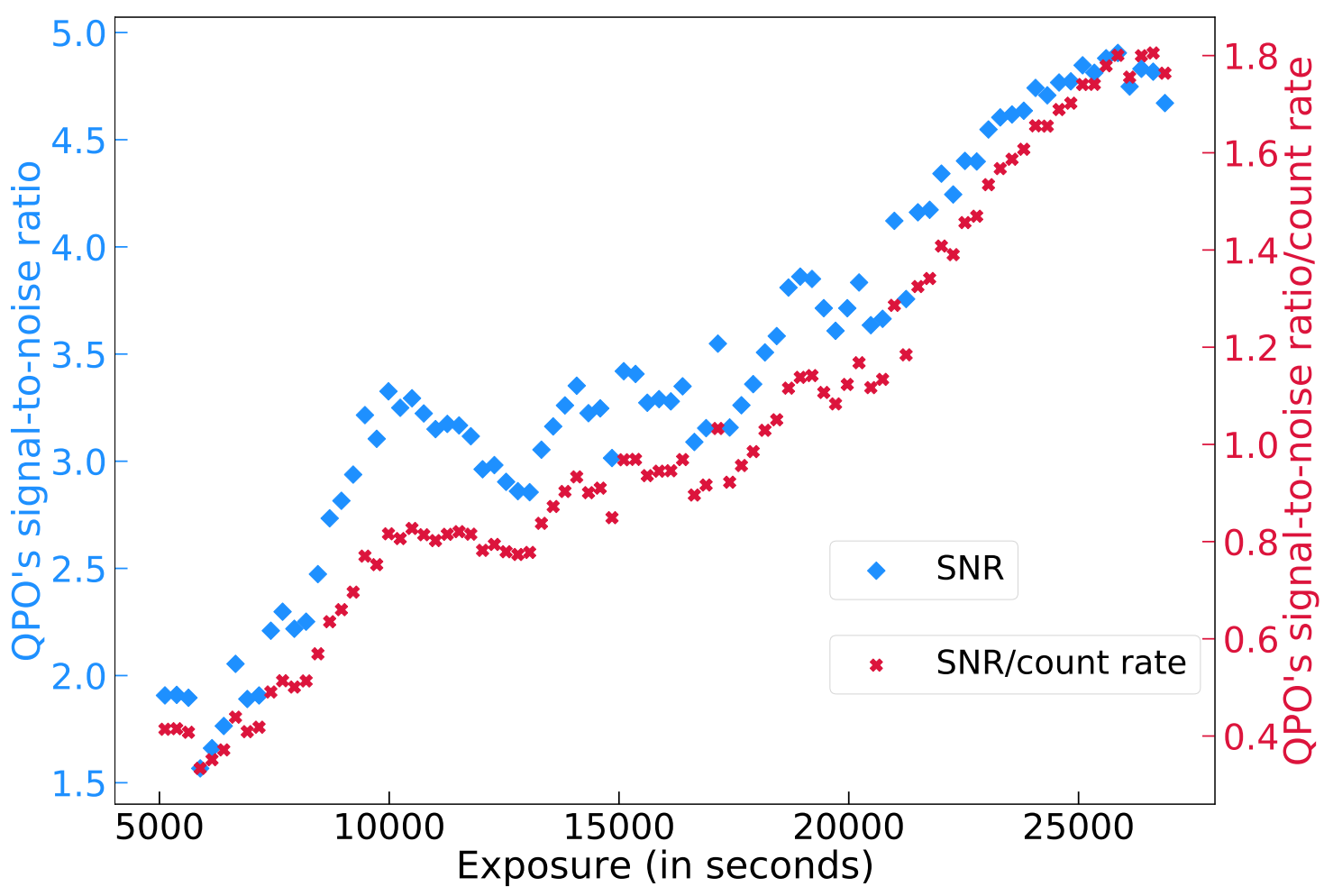

Figure S7: QPO's signal-to-noise ratio (blue diamonds) and signal-to-noise over average count rate (red crosses) vs accumulated exposure time. It is evident that the QPO's signalto-noise gradually increases with increasing exposure. This suggests that the signal is persistent throughout the NICER monitoring period and it does not originate from any single exposure. The steepening of the mean slope around 20000 s corresponds to day 17. Note that the data points are not independent (see sec. 2.4 for more details). See also supplement movie S1. Data available in supplementary files. 
700 Movie S1: The top panel of the movie shows the evolution of the average PDS and the gradual 701 improvement in the QPO signal at $225 \mathrm{~Hz}$ with accumulated exposure. The lower panel shows 702 the corresponding long-term light curve. The shaded red rectangle in the top panel shows the 703 location of the $225 \mathrm{~Hz}$ QPO. This suggests that the QPO is long-lived and present in majority 704 of the observations. This is available as Movie_S1.gif. 


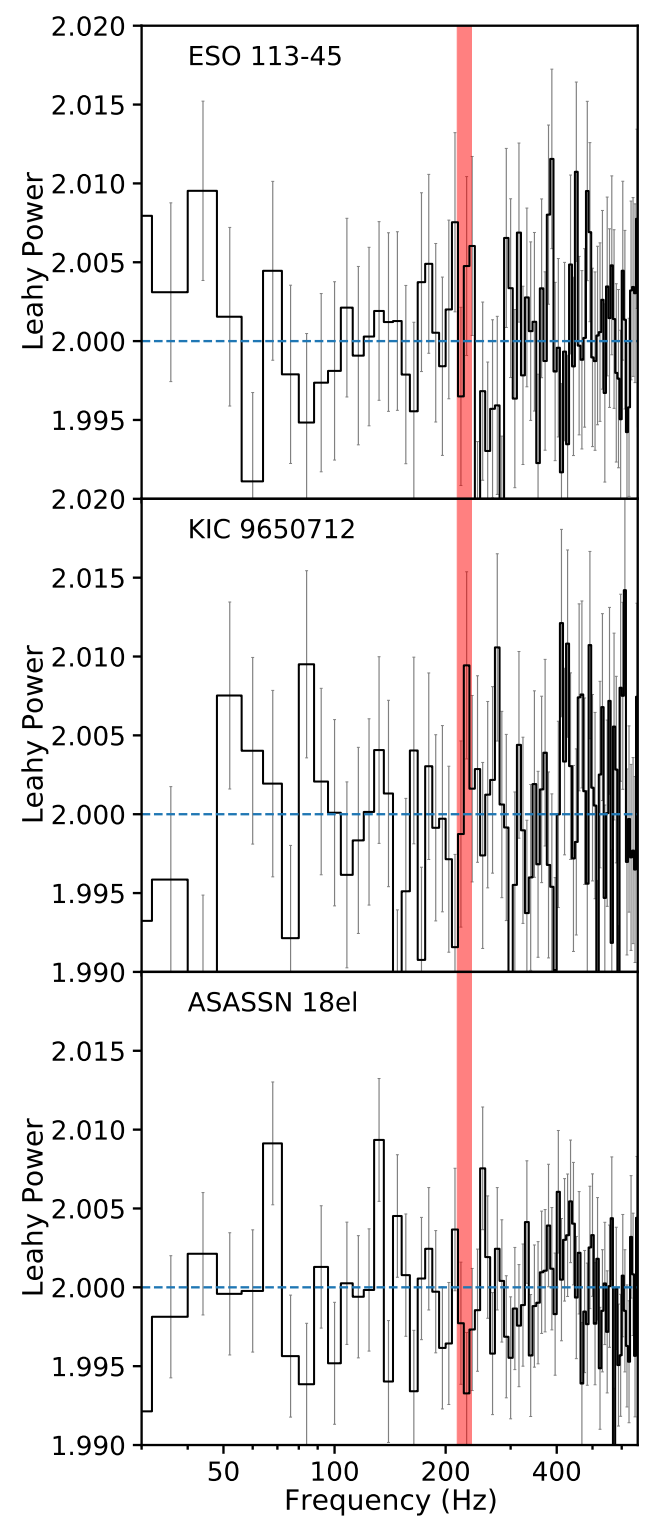

Figure S8: Average PDS of 3 AGN monitored by NICER during the same 2 month period as AT2018cow. The AGN names are shown on each panel. The mean count rates (exposures) (from top to bottom) are $30.5(15.6 \mathrm{ks}), 3.9(14.3 \mathrm{ks})$ and $1.2(33.3 \mathrm{ks})$ counts/s, respectively. These PDS were extracted exactly the same way as the average PDS of AT2018cow in Fig. 1. The location of AT2018cows QPO is indicated by the red shaded area. There is no evidence for a $225 \mathrm{~Hz}$ QPO in any of these power spectra. This provides further support that the $225 \mathrm{~Hz}$ QPO in Fig. 1 is intrinsic to AT2018cow's data. 
Figures

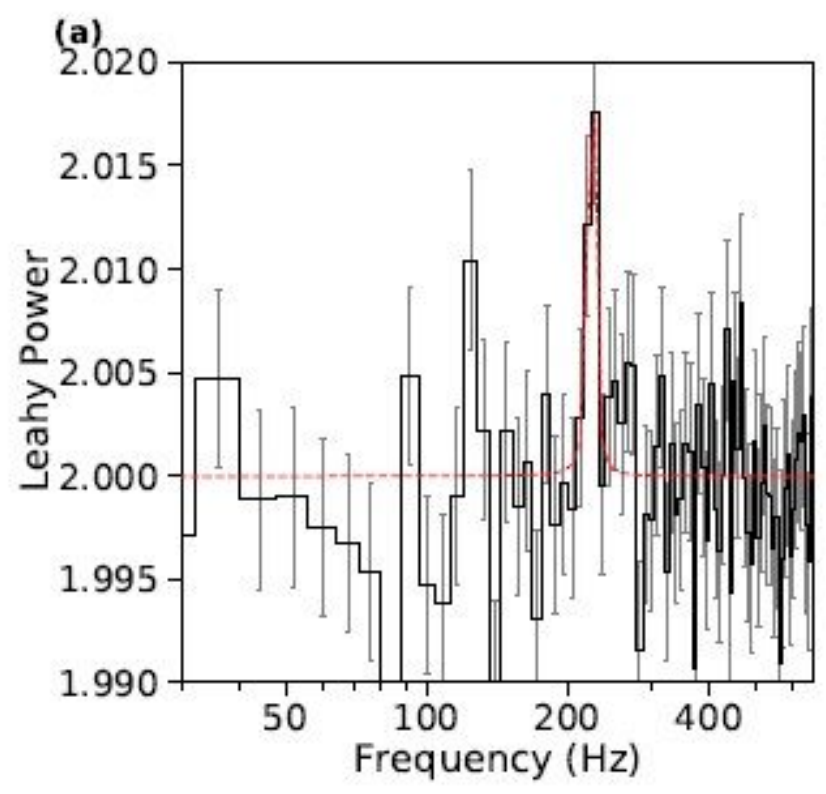

Figure 1

(see Manuscript file for figure caption) (b)

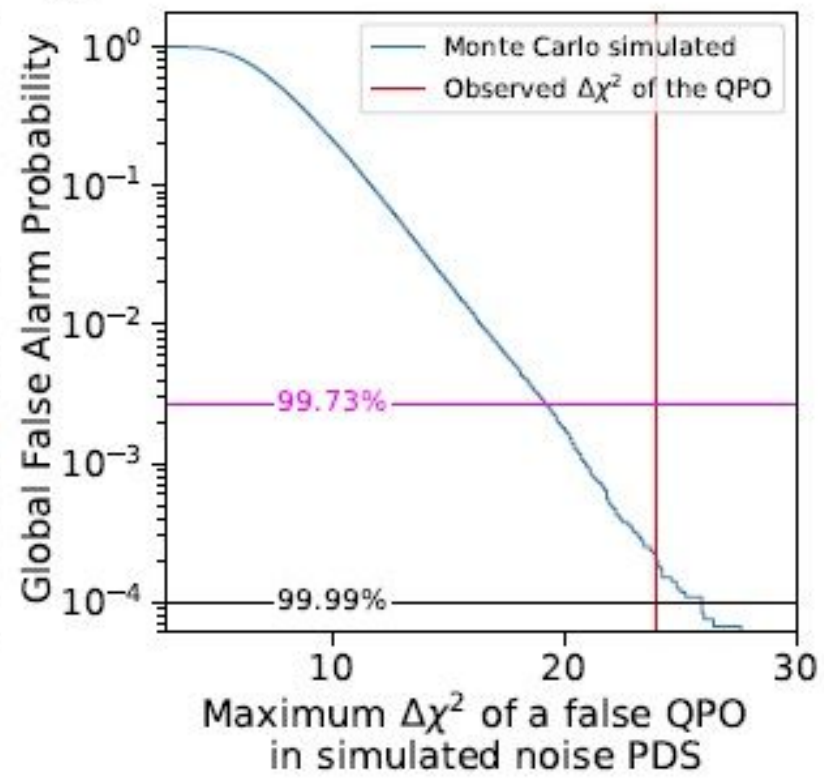




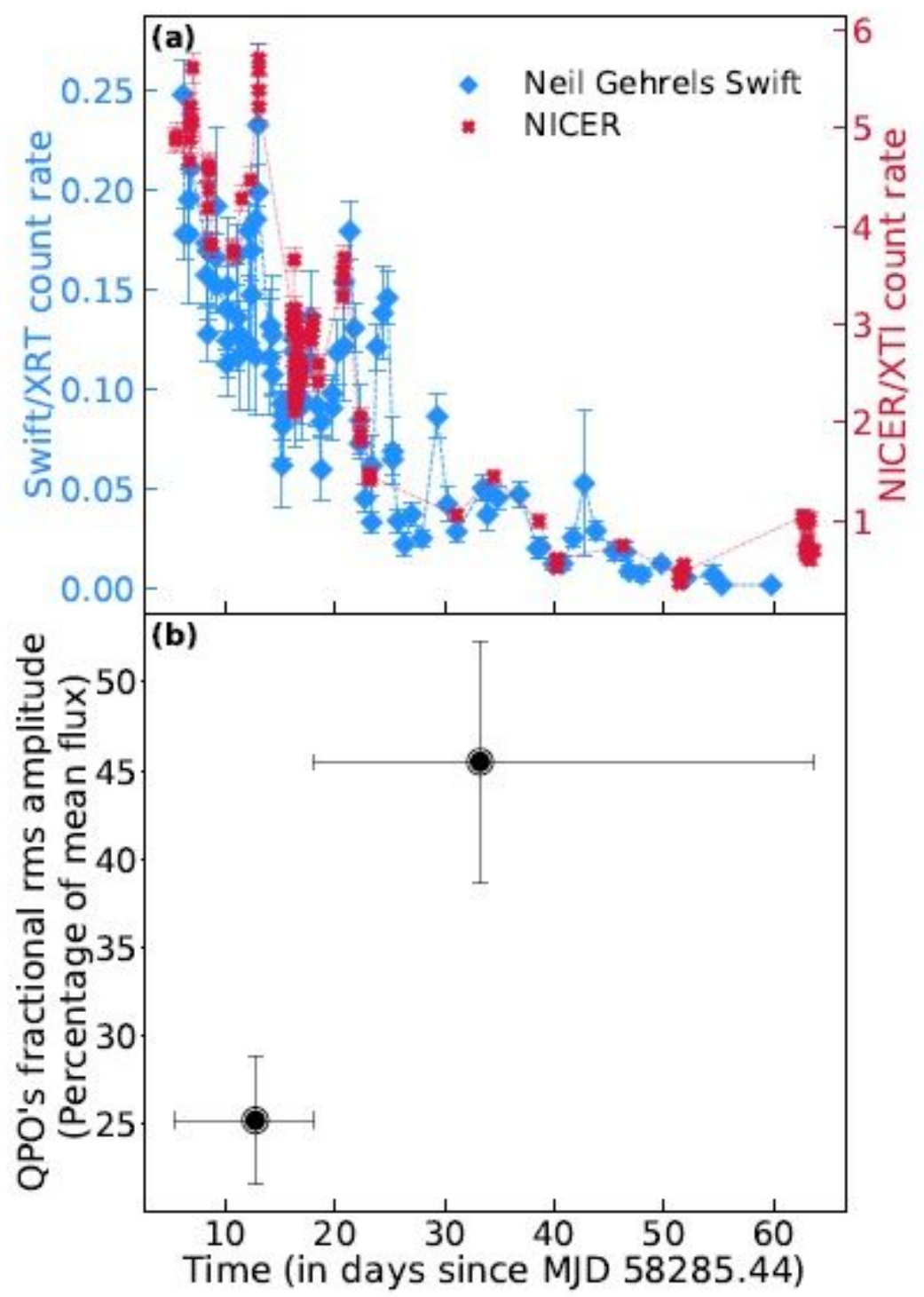

Figure 2

(see Manuscript file for figure caption)

(a)

Late Time XMM (MOS1) Image

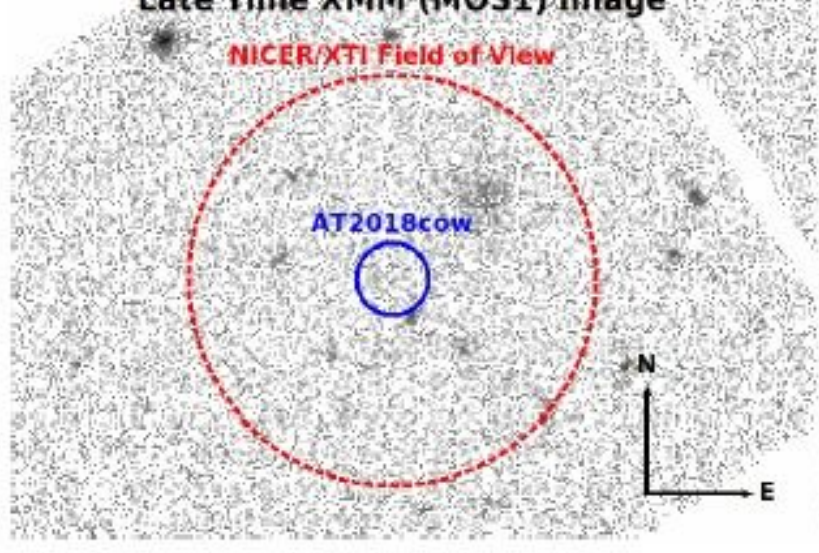

(b)

Stacked XRT Image

NICER/XTI Field of View

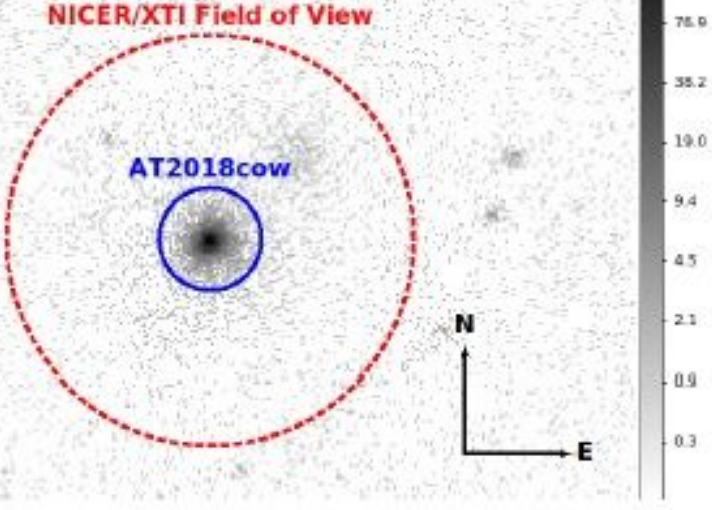


Figure 3

(see Manuscript file for figure caption)

\section{Supplementary Files}

This is a list of supplementary files associated with this preprint. Click to download.

- MovieS1.gif 